\title{
A PULSATION SEARCH AMONG YOUNG BROWN DWARFS AND VERY-LOW-MASS STARS
}

\author{
Ann Marie Cody ${ }^{1,2}$ and Lynne A. Hillendrand ${ }^{1}$ \\ ${ }^{1}$ California Institute of Technology, Department of Astrophysics, MC 249-17, Pasadena, CA 91125, USA; amc@ipac.caltech.edu \\ ${ }^{2}$ Spitzer Science Center, California Institute of Technology, 1200 East California Boulevard, Pasadena, CA 91125, USA \\ Received 2014 September 10; accepted 2014 October 17; published 2014 November 14
}

\begin{abstract}
In 2005, Palla \& Baraffe proposed that brown dwarfs (BDs) and very-low-mass stars (VLMSs; $<0.1$ solar masses) may be unstable to radial oscillations during the pre-main-sequence deuterium burning phase. With associated periods of one to four hours, this potentially new class of pulsation offers unprecedented opportunities to probe the interiors and evolution of low-mass objects in the 1-15 million year age range. Following up on reports of short-period variability in young clusters, we designed a high-cadence photometric monitoring campaign to search for deuterium-burning pulsation among a sample of 348 BDs and VLMSs in the four young clusters $\sigma$ Orionis, Chamaeleon I, IC 348, and Upper Scorpius. In the resulting light curves we achieved sensitivity to periodic signals of amplitude several millimagnitudes, on timescales from 15 minutes to two weeks. Despite the exquisite data quality, we failed to detect any periodicities below seven hours. We conclude that D-burning pulsations are not able to grow to observable amplitudes in the early pre-main sequence. In spite of the nondetection, we did uncover a rich set of variability behavior — both periodic and aperiodic — on day to week timescales. We present new compilations of variable sources from our sample, as well as three new candidate cluster members in Chamaeleon I.
\end{abstract}

Key words: brown dwarfs - open clusters and associations: individual (Chamaeleon I, IC 348, $\sigma$ Orionis, Upper Scorpius) - stars: low-mass - stars: oscillations - stars: variables: general

Online-only material: color figures

\section{PULSATION IN YOUNG STARS AND BROWN DWARFS}

Investigation of the interiors and evolutionary states of stars has long been a challenging task. Measurements of mass and age rely heavily on theoretical models, many of which require further calibration. This situation has begun to improve dramatically with the rise of asteroseismology. Precision measurements of stellar oscillation frequencies and their differences can furnish ages and masses to better than $10 \%$ (e.g., Lebreton \& Goupil 2014), and even internal rotation profiles (e.g., Kurtz et al. 2014; Beck et al. 2012).

One of the obvious limitations of asteroseismology is that it applies only to stars that are unstable to pulsation. Traditionally this has omitted most young and low-mass stars. Recently, the first pre-main sequence (PMS) pulsating stars at intermediate masses have been identified and characterized in young open clusters (Zwintz 2008; Guenther et al. 2009). These PMS $\delta$ Scuti stars inhabit an instability strip in the Hertzsprung-Russell (H-R) diagram comprising spectral types $\mathrm{A}$ to $\mathrm{F}\left(M \sim 1.5-3 M_{\odot}\right)$. Observations of their pulsations have now been used to demonstrate a relationship between oscillation frequencies and evolutionary status (Zwintz et al. 2014).

For stars less than $1.5 M_{\odot}$, asteroseismology has yet to yield fruit. Brown dwarfs (BDs) and very-low-mass stars (VLMSs) have been predicted to undergo a pulsation instability fueled by central deuterium burning (e.g., Palla \& Baraffe 2005, hereafter PB05). The pulsation theory involves destabilization via the epsilon mechanism-a conversion of nuclear energy to kinetic oscillations. In brief, their main predictions are that deuterium burning BDs and VLMSs from $\sim 0.02$ to $0.1 M_{\odot}$ will exhibit radial oscillations with periods from one to four hours. The amplitudes are indeterminate, but the PB05 calculations indicate that pulsations will grow exponentially with time if they are not damped in the stellar interior by, e.g., convection.
They argue that the mismatch between the pulsation timescale and the convective overturn timescale $(\sim$ weeks, apart from the surface layers where it is shorter) rules out the damping scenario. Empirical verification of this instability theory through detection of short-timescale periodic variability presents a new opportunity to probe the interior and surface properties of young low-mass objects. In support of the pulsation hypothesis are several previous claims of short-period variability with reported flux changes at the few to five percent level (e.g., Scholz \& Eislöffel 2004; Bailer-Jones \& Mundt 2001; Zapatero Osorio et al. 2003).

To verify or refute these findings, we initiated a pulsation search in the $\sigma$ Orionis cluster (Cody \& Hillenbrand 2010, 2011), where we encountered over 100 periodic and aperiodic variables. The conclusion of that work was that none of the light curves exhibited behavior consistent with deuterium burning oscillations on few-hour timescales. We now report the result of an expanded campaign in three other young starforming regions: IC 348, Chamaeleon I, and the Upper Scorpius association. The expanded sample enables better coverage of parameter space in the H-R diagram, as well as improved statistical constraint on pulsation amplitudes. In addition to searching for short-period oscillations, we have also mined these fields for longer timescale variability and new cluster members.

\section{TARGET SELECTION AND OBSERVATIONS}

The prospect of detecting pulsation in young BDs and VLMSs is dependent on the existence of a suitable observational sample with characteristics in the range of the prospective pulsators. Because the pulsation instability strip calculated by PB05 is fairly narrow compared to the characteristic range of luminosities observed in 1-10 Myr clusters, most very low mass members of a young, roughly coeval population may not be expected to pulsate. Yet substantial uncertainties in luminosity 
Table 1

Photometric Observations Comprising the Pulsation Search Campaign

\begin{tabular}{|c|c|c|c|c|c|c|c|c|}
\hline Cluster & Telescope & Instrument & $\begin{array}{l}\text { Field center } \\
\text { (R.A., Decl.) }\end{array}$ & FOV & Dates & $\begin{array}{l}\text { Duty } \\
\text { cycle }\end{array}$ & $\begin{array}{l}\text { Cadence } \\
\text { (seconds) }\end{array}$ & Band \\
\hline$\sigma$ Ori & CTIO $1.0 \mathrm{~m}$ & Y4KCam & $5: 38: 00.6,-02: 43: 44$ & $20^{\prime} \times 20^{\prime}$ & 2007 Dec 27-2008 Jan 7, & $25 \%$ & 460 & $\mathrm{I}, \mathrm{R}$ \\
\hline Cha I & CTIO $1.0 \mathrm{~m}$ & Y4KCam & 11:09:51.0, -77:27:44 & $20^{\prime} \times 20^{\prime}$ & 2008 May $13-25$ & $25 \%$ & 700 & $\mathrm{i}, \mathrm{r}$ \\
\hline USco & CTIO $1.0 \mathrm{~m}$ & Y4KCam & $16: 11: 08,-22: 12: 04$ & $20^{\prime} \times 20^{\prime}$ & 2008 May $13-16 ; 21-22$ & $15 \%$ & 700 & $\mathrm{i}, \mathrm{r}$ \\
\hline USco & CTIO $1.0 \mathrm{~m}$ & Y4KCam & $16: 17: 57.5,-23: 45: 41$ & $20^{\prime} \times 20^{\prime}$ & 2008 May 23-25 & $15 \%$ & 700 & $\mathrm{i}, \mathrm{r}$ \\
\hline USco & P60 & (CCD) & $16: 13: 17.5,-19: 27: 00$ & $12^{\prime} .5 \times 12^{\prime} .5$ & 2008 Jun $1-14$ & $13 \%$ & 330 & ip \\
\hline IC 348 & P60 & (CCD) & $3: 44: 21.8,+32: 05: 43$ & $12 \prime .5 \times 12^{\prime} .5$ & 2008 Nov $17-23$, Nov $28-29$ & $18 \%$ & 270 & ip, $\mathrm{Cr}$ \\
\hline$\sigma$ Ori & CTIO $1.0 \mathrm{~m}$ & Y4KCam & $5: 39: 31.1,-02: 37: 26$ & $20^{\prime} \times 20^{\prime}$ & 2008 Dec $14-24$ & $28 \%$ & 700 & I, R \\
\hline USco & P60 & (CCD) & $16: 17: 46.3,-20: 54: 18$ & $12^{\prime} .5 \times 12^{\prime} .5$ & 2009 May $14-30$ & $13 \%$ & 330 & ip, rp \\
\hline$\sigma$ Ori & Spitzer & IRAC & $05: 38: 23.3,-02: 40: 29$ & $5^{\prime} .2 \times 5^{\prime} \cdot 2$ & 2009 Oct $22-23$ & $100 \%$ & 31 & $3.6 \mu \mathrm{m}$ \\
\hline$\sigma$ Ori & Spitzer & IRAC & $05: 38: 26.4,-02: 47: 13$ & $5^{\prime} .2 \times 5^{\prime} .2$ & 2009 Oct $22-23$ & $100 \%$ & 31 & $4.5 \mu \mathrm{m}$ \\
\hline IC 348 & $H S T$ & WFC3 & $03: 44: 19.5,+32: 06: 20$ & $162^{\prime \prime} \times 81^{\prime \prime}$ & 2011 Jan 29-Feb 4 & $47 \%, 30 \%{ }^{a}$ & 400 & F814W \\
\hline
\end{tabular}

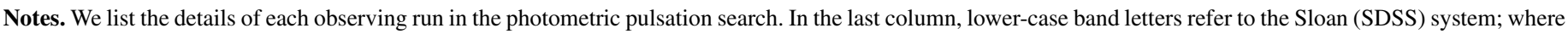

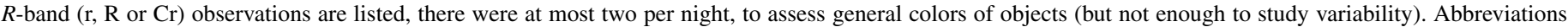

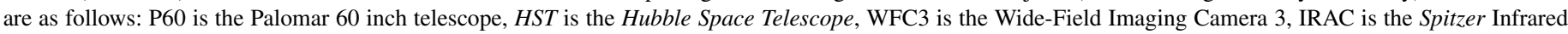

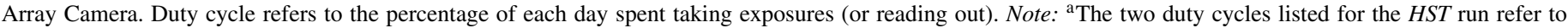

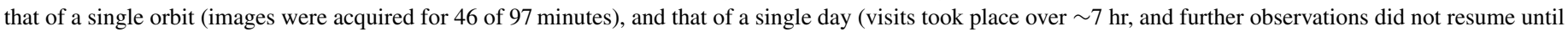
approximately one day later).

estimates and theoretical assumptions preclude determination of exactly which objects should display the phenomenon. To harvest enough candidate pulsators for statistical evaluation, our survey therefore relied on a large sample size. Selection of suitable clusters for the campaign involved two requirements: (1) that the known young member population extend into the substellar regime where pulsation is predicted to occur, and (2) that the members be bright enough for high-precision photometry with telescope apertures of up to a few meters. This limited the cluster distance to $500 \mathrm{pc}$. The level of extinction in some star-forming regions further restricted the number of available targets.

To assemble a list of pulsation candidates according to the above criteria, we relied on the substantial populations of VLMS and $\mathrm{BD}$ deuterium burning objects that have been identified in open clusters and star forming regions. Most cluster surveys to date have selected candidates based on photometric colors and confirmed membership by identifying features of youth (e.g., strong $\mathrm{H} \alpha$ emission or low-gravity lines) in follow-up spectra. However, the rich variety of phenomena in these regions, as traced by X-ray activity, photometric variability, accretion, and circumstellar disk signatures, provides alternative methods for uncovering young stellar and substellar objects. We compiled a list of the young clusters and associations with VLMSs and BDs; the regions chosen for study were $\sigma$ Orionis, Chamaeleon I, Upper Scorpius, and IC 348. Ultimately, our multi-telescope monitoring program covered a significant fraction of the spectroscopically confirmed very-low-mass objects in these young star-forming areas. The 1-10 Myr range in median ages of these clusters enables testing the effect of not only mass, but also age, on our results.

To choose specific BDs and VLMSs suitable for the photometric monitoring campaign, we focused on targets with a high probability of exhibiting pulsation, based on position relative to PB05's pulsation instability strip on the H-R diagram. For most of the clusters, it was crucial to optimize the field positions to include as many pulsation candidates as possible. This task was initiated by searching the literature relevant to each of the four chosen clusters for spectral types later than M4 (corresponding to $\lesssim 0.2 M_{\odot}$, and a high likelihood that they are still burning deuterium) as well as established membership, to rule out status as field dwarfs. The collected spectral types and brightnesses were then used to produce H-R diagrams and compare the positions of known BDs and VLMSs with the deuterium-burning instability strip. We detail the selected targets and the suite of observations conducted for each region below; a summary is provided in Table 1.

\section{1. $\sigma$ Orionis}

The $\sigma$ Orionis cluster was our primary target in the search for pulsation, with observations of candidate very-low-mass members in three fields. At approximately $3 \mathrm{Myr}$ of age (Sherry et al. 2008), it should contain numerous stars and BDs that are still burning deuterium. Two $20^{\prime} \times 20^{\prime}$ fields were initially selected for monitoring with the Cerro Tololo Inter-American Observatory $1.0 \mathrm{~m}$ telescope ("CTIO $1.0 \mathrm{~m}$ ") from 2007 December 27 to 2008 January 7 and from 2008 December 14 to 24 . We identified VLMSs and BDs in the cluster by mining the works of Béjar et al. (1999, 2001), Barrado y Navascués et al. (2001, 2003), Béjar et al. (2004), Caballero et al. (2004), Sherry et al. (2004), Scholz \& Eislöffel (2004), Burningham et al. (2005), Kenyon et al. (2005), Franciosini et al. (2006), Caballero et al. (2007), Hernández et al. (2007), Caballero (2008), Luhman et al. (2008), and Lodieu et al. (2009); a total of 153 confirmed and candidate $\sigma$ Orionis members fell in the two CTIO fields, including 15 spectroscopically confirmed young BDs. Further details on the selection, as well as a full list of objects and an image of the field of view, are provided in Cody \& Hillenbrand (2010, hereafter CH10).

We re-observed a subset of the CTIO targets in the infrared with the Spitzer Space Telescope Warm Mission in 2009, focusing on a set of five confirmed and two candidate BD members of $\sigma$ Orionis. Also included in the Spitzer 3.6 and $4.5 \mu \mathrm{m}$ fields were seven additional higher mass $\left(>0.1 M_{\odot}\right)$ cluster members. All targets were monitored continuously for a single $24 \mathrm{hr}$ period, at a cadence of $\sim 30 \mathrm{~s}$. An extensive description of these observations is available in Cody \& Hillenbrand (2011).

\subsection{Chamaeleon I}

Initial target compilation for the Chamaeleon I cluster was based on Luhman's (2007) work, plus additional members from 


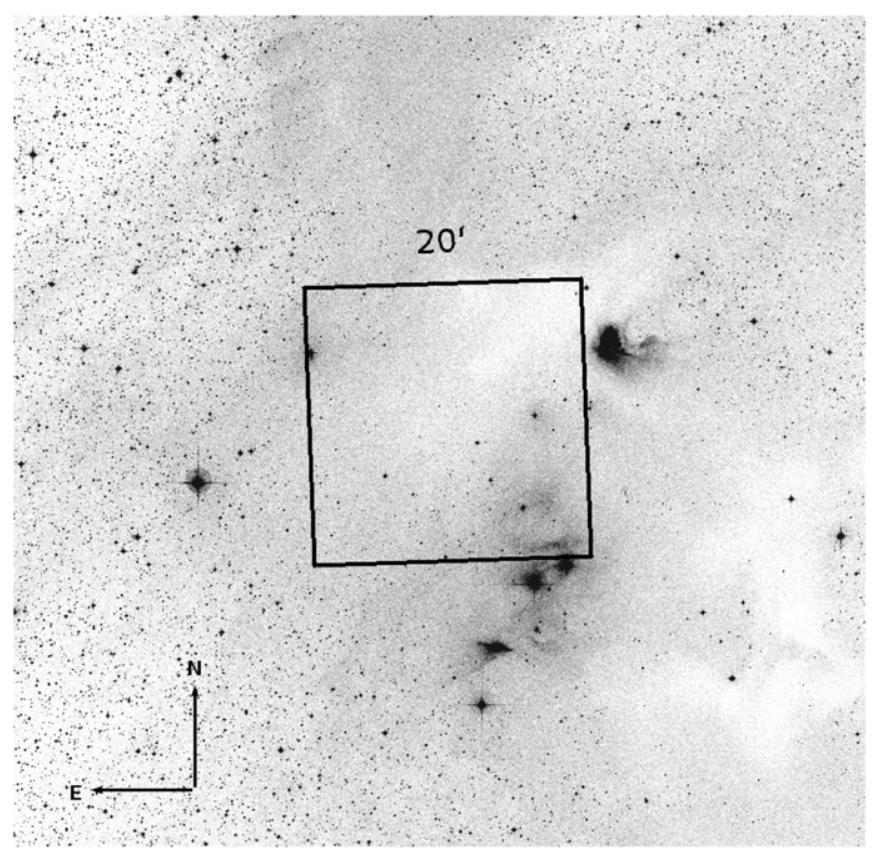

Figure 1. Cha I field observed with the CTIO $1.0 \mathrm{~m}$ telescope is shown superimposed on a Digital Sky Survey image. Extinction in this region is highly variable, and we have avoided the most nebulous dark cloud region toward the west.

Luhman \& Muench (2008), Luhman et al. (2008), and Mužić et al. (2011). These studies indicate a median age for the cluster of $\sim 2 \mathrm{myr}$, making it slightly younger than $\sigma$ Orionis, and a prime candidate for the deuterium burning pulsation search. The campaign on Cha I involved observations of a single $20^{\prime} \times$ $20^{\prime}$ field with the CTIO $1.0 \mathrm{~m}$ from 2008 May $13-25$. The field of view (FOV) was selected to maximize the number of BDs monitored and also avoid some of the dense nebulosity in this region; it is displayed in Figure 1.

From the full source compilation we selected a total of 32 Cha I members for observation, of which 6 have spectral types consistent with substellar status (spectral type M6 and later) and 22 more are likely VLMSs with (M4 or later). The remaining four are higher mass stars and not expected to pulsate. We have compiled the existing photometric and spectroscopic data on the set of 32 objects, including optical through near-infrared photometry and spectral types, in Table 2.

\subsection{IC 348}

IC 348 is an appealing target in the search for pulsation, since it is relatively compact $\left(<1^{\circ}\right.$ square $)$, and its membership is very well characterized (Luhman et al. 2003; Muench et al. 2007). At a 2-3 myr (Luhman et al. 2003), it is comparable in age to $\sigma$ Orionis and Cha I. Several previous studies have identified numerous periodic variables, which are presumably the result of rotational modulation of spots (Cohen et al. 2004; Littlefair et al. 2005; Cieza \& Baliber 2006, and references therein). The typical periods are near two to three days, but several objects have reported periods as short as five hours.

We observed IC 348 from the ground with the Palomar 60 inch telescope ("P60"; Cenko et al. 2006) and from space using the Hubble Space Telescope (HST) Wide Field Camera 3 (WFC3). The $\sim 12$ '.5 $\times 12$ '.5 ground-based field of view encompassed a significant spatial extent within IC 348, including the nebulous region in the cluster center. The WFC 3 field is much smaller, with a full field of view of $162^{\prime \prime} \times 162^{\prime \prime}$. To maximize the data

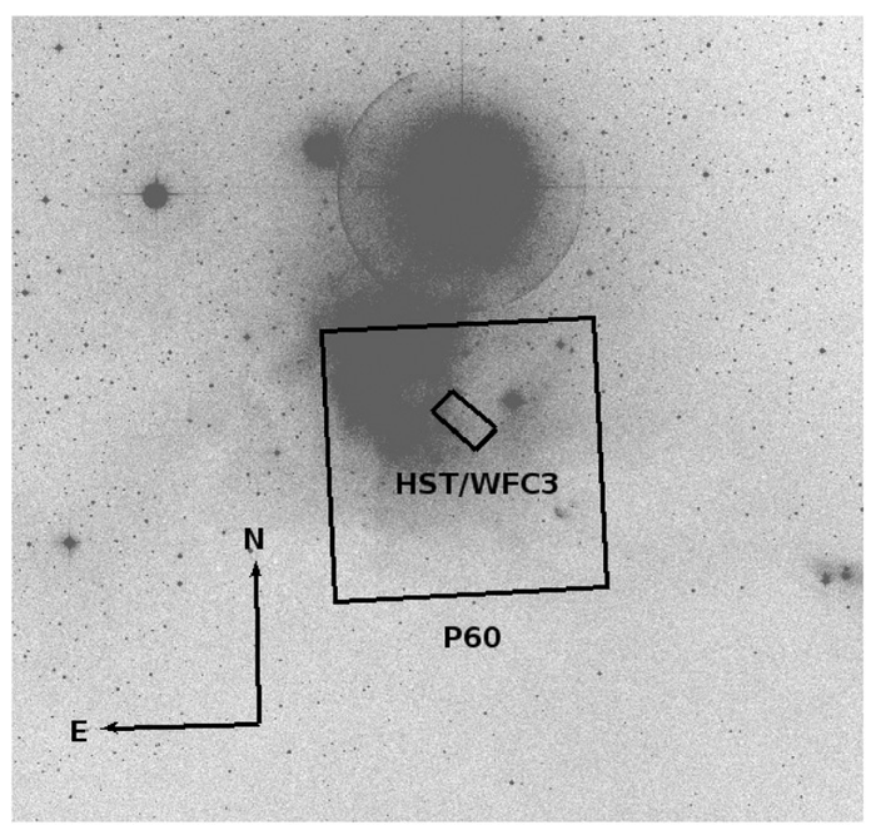

Figure 2. IC 348 fields observed with the Palomar 60 inch telescope $(12.5 \times$ $\left.12^{\prime} .5\right)$ and $H S T\left(81^{\prime \prime} \times 162^{\prime \prime}\right)$ are shown superimposed on a Digital Sky Survey image. The bright B star binary o Per lies just to the north.

cadence for $H S T$ we opted to observe in the subarray mode, for which only one of two $81^{\prime \prime} \times 162^{\prime \prime}$ chips was used. Since the ground-based photometry preceded the space observations by more than three years, we were able to select several faint BD pulsation candidates that required photometry at the higher sensitivity levels afforded by HST. Therefore the WFC3 field did not cover an additional region, but rather fell within the previous ground-based FOV, encompassing four BDs and and two VLMSs. Two of these (L761 and L1434) do not have ground-based light curves since they suffered from low signalto-noise. Both the ground- and space-based fields are illustrated in Figure 2.

Selection of low-mass IC 348 cluster objects was carried out by considering the spectral types presented by (Luhman et al. 2003, 2005). A total of 194 members fell within the chosen ground-based FOV. However, we did not extract photometry for stars that fell on bad pixel columns, or for some BDs that were too faint for adequate signal to noise. There are also a number of brighter stars within the field for which we did not obtain data since their point-spread functions (PSFs) were saturated and distorted by nebulosity and scattered light within the central region of the cluster where several bright B stars lie. For the pulsation campaign, we ultimately monitored 147 low-mass IC 348 members, including $26 \mathrm{BDs}$ (spectral types M6 or later) and 65 VLMSs (M4-M6). We present a compilation of basic target properties in Tables 3 and 4.

\subsection{Upper Scorpius}

The Upper Scorpius (USco) region is one of the most spatially extended young associations, with stars and BDs spread over many tens of degrees on the sky. As a result, few variability studies have been performed here, apart from the work of Slesnick (2008). It is significantly older than the other three regions studied, with age estimates from 5 to 11 myr (Preibisch et al. 2002; Pecaut et al. 2012). Nevertheless, an examination of the H-R diagram of catalogued low-mass members shows that 
Table 2

Cha I Objects Observed

\begin{tabular}{|c|c|c|c|c|c|c|}
\hline Object & Other ID & $\mathrm{SpT}$ & $i$ & $J$ & $H$ & $K_{s}$ \\
\hline 2MASS J11105076-7718031 & ESO Halpha 568 & M4.25 & 14.38 & $12.044 \pm 0.023$ & $11.101 \pm 0.023$ & $10.748 \pm 0.024$ \\
\hline 2MASS J11105359-7725004 & ISO 256 & M4.5 & 17.51 & $14.271 \pm 0.030$ & $12.507 \pm 0.027$ & $11.339 \pm 0.021$ \\
\hline 2MASS J11065906-7718535 & ISO 89 & M4.25 & 12.97 & $11.204 \pm 0.026$ & $10.423 \pm 0.021$ & $10.003 \pm 0.024$ \\
\hline 2MASS J11070925-7718471 & ISO 91 & M3 & $\cdots$ & $14.902 \pm 0.042$ & $12.581 \pm 0.021$ & $11.476 \pm 0.023$ \\
\hline 2MASS J11071668-7735532 & Cha Halpha 1 & M7.75 & 16.38 & $13.342 \pm 0.024$ & $12.668 \pm 0.026$ & $12.174 \pm 0.024$ \\
\hline 2MASS J11071860-7732516 & Cha Halpha 9 & M5.5 & $\ldots$ & $13.733 \pm 0.026$ & $12.492 \pm 0.023$ & $11.803 \pm 0.024$ \\
\hline 2MASS J11072040-7729403 & ISO 99 & M4.5 & 13.00 & $11.134 \pm 0.024$ & $10.547 \pm 0.023$ & $10.259 \pm 0.021$ \\
\hline 2MASS J11073519-7734493 & CHXR 76 & M4.25 & 14.39 & $12.127 \pm 0.023$ & $11.279 \pm 0.023$ & $10.954 \pm 0.021$ \\
\hline 2MASS J11073686-7733335 & CHXR 26 & M3.5 & 15.18 & $11.593 \pm 0.030$ & $10.045 \pm 0.035$ & $9.348 \pm 0.027$ \\
\hline 2MASS J11073775-7735308 & Cha Halpha 7 & M7.75 & 17.03 & $13.613 \pm 0.030$ & $12.900 \pm 0.026$ & $12.421 \pm 0.030$ \\
\hline 2MASS J11074245-7733593 & Cha Halpha 2 & M5.25 & 15.26 & $12.210 \pm 0.024$ & $11.243 \pm 0.026$ & $10.675 \pm 0.021$ \\
\hline 2MASS J11075225-7736569 & Cha Halpha 3 & M5.5 & 15.07 & $12.292 \pm 0.024$ & $11.520 \pm 0.023$ & $11.097 \pm 0.019$ \\
\hline 2MASS J11081850-7730408 & ISO 138 & M6.5 & 16.77 & $14.057 \pm 0.030$ & $13.466 \pm 0.035$ & $13.040 \pm 0.032$ \\
\hline Cha J11081938-7731522 & $\ldots$ & M4.75 & $\ldots$ & $\ldots$ & $\ldots$ & $\ldots$ \\
\hline 2MASS J11082238-7730277 & ISO 143 & M5 & 15.51 & $12.570 \pm 0.024$ & $11.651 \pm 0.027$ & $11.095 \pm 0.023$ \\
\hline 2MASS J11083952-7734166 & Cha Halpha 6 & M5.75 & 15.06 & $12.263 \pm 0.027$ & $11.479 \pm 0.024$ & $11.038 \pm 0.027$ \\
\hline 2MASS J11085421-7732115 & CHXR 78C & M5.25 & 15.01 & $12.310 \pm 0.026$ & $11.555 \pm 0.023$ & $11.224 \pm 0.024$ \\
\hline 2MASS J11085596-7727132 & ISO 167 & M5.25 & 17.08 & $13.514 \pm 0.031$ & $12.293 \pm 0.026$ & $11.619 \pm 0.025$ \\
\hline 2MASS J11093543-7731390 & $\ldots$ & M8.25 & $\cdots$ & $15.936 \pm 0.092$ & $15.022 \pm 0.087$ & $14.412 \pm 0.101$ \\
\hline 2MASS J11094260-7725578 & ISO 200 & M5 & 15.91 & $12.329 \pm 0.027$ & $11.175 \pm 0.026$ & $10.552 \pm 0.028$ \\
\hline 2MASS J11094742-7726290 & ESO Halpha 567 & M3.25 & 16.62 & $12.767 \pm 0.027$ & $11.228 \pm 0.023$ & $10.236 \pm 0.022$ \\
\hline 2MASS J11094918-7731197 & KG 102 & M5.5 & 15.64 & $13.057 \pm 0.036$ & $12.229 \pm 0.039$ & $11.802 \pm 0.034$ \\
\hline 2MASS J11095336-7728365 & ISO 220 & M5.75 & $\ldots$ & $14.300 \pm 0.039$ & $13.020 \pm 0.026$ & $12.233 \pm 0.025$ \\
\hline 2MASS J11100192-7725451 & LM04_419 & M5.25 & 17.46 & $13.833 \pm 0.032$ & $12.605 \pm 0.026$ & $12.021 \pm 0.03$ \\
\hline 2MASS J11100785-7727480 & ISO 235 & M5.5 & 17.79 & $13.545 \pm 0.030$ & $12.097 \pm 0.026$ & $11.342 \pm 0.023$ \\
\hline 2MASS J11101153-7733521 & $\cdots$ & M4.5 & 14.24 & $12.183 \pm 0.031$ & $11.192 \pm 0.023$ & $10.783 \pm 0.019$ \\
\hline 2MASS J11103481-7722053 & LM04_405 & M4 & $\ldots$ & $12.038 \pm 0.023$ & $10.718 \pm 0.024$ & $10.034 \pm 0.019$ \\
\hline 2MASS J11103644-7722131 & ISO 250 & M4.75 & 16.52 & $12.724 \pm 0.027$ & $11.369 \pm 0.026$ & $10.667 \pm 0.021$ \\
\hline 2MASS J11103801-7732399 & CHXR 47 & K3 & 11.90 & $9.741 \pm 0.027$ & $8.687 \pm 0.047$ & $8.277 \pm 0.029$ \\
\hline 2MASS J11104141-7720480 & ISO 252 & M6 & 17.29 & $13.860 \pm 0.030$ & $12.891 \pm 0.027$ & $12.266 \pm 0.023$ \\
\hline 2MASS J11120288-7722483 & $\cdots$ & M6 & $\cdots$ & $13.588 \pm 0.030$ & $12.941 \pm 0.044$ & $12.510 \pm 0.030$ \\
\hline 2MASS J11120351-7726009 & ISO 282 & M4.75 & $\cdots$ & $13.626 \pm 0.024$ & $12.587 \pm 0.025$ & $11.842 \pm 0.023$ \\
\hline
\end{tabular}

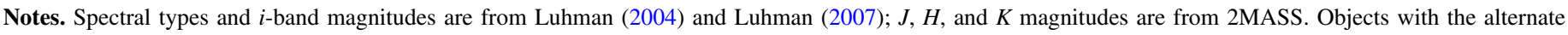
identification LM04 are from the catalog of Martí et al. (2004).

many objects have temperatures and luminosities overlapping the D-burning instability strip.

Because of the sparseness of this association, it is difficult to obtain data on more than one target at a time. We therefore selected fields carefully to maximize the number of pulsation candidates. We ultimately observed five different fields in Upper Scorpius, including 5 BDs and 11 VLMSs, with identifications and references listed in Table 5. None of these regions contained any nebulosity. Observations of three of the fields were abbreviated to three nights or less because of weather (the CTIO $1.0 \mathrm{~m}$ run), and tracking problems (USco members in the first field chosen for observation with the P60 fell too close to the edge of the detector and tended to wander out of the FOV). The list of dates is provided in Table 1. Since our observations in 2008 and 2009, further low-mass Upper Scorpius members have been discovered by Lodieu et al. (2011) and Dawson et al. (2011). We identified three of these objects from Lodieu et al. (2011) in our first FOV from CTIO $1.0 \mathrm{~m}$ Y4KCam monitoring in 2008 May, with no additional targets in any of the other observations.

\section{DATA ACQUISITION AND REDUCTION}

We employed four different telescopes in the search for pulsation, as summarized in Table 1. An extensive discussion of observations with the Spitzer Space Telescope is provided in
Cody \& Hillenbrand (2011); here we detail the observing setups with the other three facilities.

\subsection{Cerro Tololo Inter-American Observatory $1.0 \mathrm{~m}$ Telescope}

We observed low-mass targets in $\sigma$ Orionis, Chamaeleon I, and Upper Scorpius with the CTIO $1.0 \mathrm{~m}$. The observational setup and data reduction procedures were the nearly same for all CTIO $1.0 \mathrm{~m}$ runs, and they are described in detail in $\mathrm{CH} 10$. The selected exposure time was $600 \mathrm{~s}$ for all runs, apart from the first set of observations on $\sigma$ Orionis members (360 s).

The main distinction for the 2008 May observing run (see Table 1) was that sky conditions were not photometric, and just over two nights were lost to clouds. In addition, telescope building maintenance caused a new accumulation of dust specks on the detector each night, which resulted in inconsistent sky flatfield acquisition. We acquired dome flatfields at the beginning and end of each night to calibrate out dust "donuts," but these dome flats are known to misrepresent the true pixel sensitivity distribution by up to $10 \%$. Therefore, we carried out flatfielding with sky flats on nights where at least seven were available, and when clouds precluded the acquisition of sky flats, we instead relied on the dome flats but performed an illumination correction using the high signal-to-noise composite provided by P. Massey. ${ }^{3}$

\footnotetext{
3 http://www.lowell.edu/users/massey/obins/y4kcamred.html
} 
Table 3

IC 348 Cluster Members Observed with the Palomar 60 inch Telescope

\begin{tabular}{|c|c|c|c|c|c|c|}
\hline Object & 2MASS ID & $I$ & $J$ & $H$ & $\mathrm{SpT}$ & Object \\
\hline L13 & 2MASS J03435964+3201539 & 19.46 & 13.45 & 10.78 & M0.5 & L199 \\
\hline L23 & 2MASS J03443871+3208420 & 13.97 & 11.19 & 9.97 & $\mathrm{~K} 3$ & L203 \\
\hline L26 & 2MASS J03435602+3202132 & 15.62 & 12.29 & 10.56 & K7 & L205 \\
\hline L31 & 2MASS J03441816+3204570 & 15.37 & 12.09 & 10.54 & G1 & L207 \\
\hline L32 & 2MASS J03443788+3208041 & 14.18 & 11.69 & 10.48 & K7 & L210 \\
\hline L35 & 2MASS J03443924+3207355 & 13.21 & 10.83 & 9.95 & $\mathrm{~K} 3$ & L215 \\
\hline L37 & 2MASS J03443798+3203296 & 13.18 & 11.45 & 10.44 & K6 & L217 \\
\hline L40 & 2MASS J03442972+3210398 & 14.10 & 11.93 & 10.76 & K8 & L221 \\
\hline L41 & 2MASS J03442161+3210376 & 14.99 & 12.49 & 11.28 & K7 & L228 \\
\hline L46 & 2MASS J03441162+3203131 & 16.24 & 12.78 & 11.22 & G8 & L230 \\
\hline L48 & 2MASS J03443487+3206337 & 13.45 & 11.50 & 10.60 & K5.5 & L234 \\
\hline L49 & 2MASS J03435759+3201373 & 19.63 & 14.56 & 11.89 & M0.5 & L237 \\
\hline L51 & 2MASS J03441297+3201354 & 19.56 & 15.09 & 12.43 & $\ldots$ & L243 \\
\hline L52 & 2MASS J03444351+3207427 & 14.98 & 12.12 & 10.89 & M1 & $\mathrm{L} 252$ \\
\hline L55 & 2MASS J03443137+3200140 & 18.06 & 13.63 & 11.65 & M0.5 & L253 \\
\hline L56 & 2MASS J03440499+3209537 & 13.02 & 11.55 & 10.71 & K3.5 & L254 \\
\hline L58 & 2MASS J03443854+3208006 & 14.24 & 11.94 & 10.90 & M1.25 & L255 \\
\hline L61 & 2MASS J03442228+3205427 & 15.23 & 12.54 & 11.27 & K8 & L256 \\
\hline L65 & 2MASS J03443398+3208541 & 13.69 & 11.85 & 10.98 & M0 & L259 \\
\hline L66 & 2MASS J03442847+3207224 & 13.53 & 11.67 & 10.85 & K6.5 & L266 \\
\hline L68 & 2MASS J03442851+3159539 & 14.16 & 12.00 & 11.13 & M3.5 & L276 \\
\hline L69 & 2MASS J03442702+3204436 & 13.69 & 11.95 & 11.14 & M1 & L277 \\
\hline L71 & 2MASS J03443257+3208558 & 14.32 & 12.11 & 11.13 & M3 & L278 \\
\hline L72 & 2MASS J03442257+3201536 & 14.31 & 12.12 & 11.15 & $\mathrm{M} 2.5$ & L287 \\
\hline L74 & 2MASS J03443426+3210497 & 14.36 & 12.14 & 11.13 & M2 & L298 \\
\hline L75 & 2MASS J03444376+3210304 & 14.26 & 12.75 & 11.60 & M1.25 & L300 \\
\hline L82 & 2MASS J03443740+3206118 & 13.89 & 12.09 & 11.15 & K7 & L301 \\
\hline L83 & 2MASS J03443741+3209009 & 14.93 & 12.49 & 11.44 & M1 & L302 \\
\hline L91 & 2MASS J03443919+3209448 & 14.76 & 12.59 & 11.52 & M2 & L303 \\
\hline L92 & 2MASS J03442366+3206465 & 14.20 & 12.24 & 11.37 & M2.5 & L308 \\
\hline L97 & 2MASS J03442554+3206171 & 15.98 & 12.82 & 11.59 & M2.25 & L312 \\
\hline L98 & 2MASS J03443860+3205064 & 14.88 & 12.47 & 11.52 & M4 & L314 \\
\hline L99 & $\cdots$ & 14.78 & 12.89 & 11.90 & M3.75 & L322 \\
\hline L103 & 2MASS J03444458+3208125 & 15.73 & 12.89 & 11.87 & M2 & L324 \\
\hline L105 & 2MASS J03441125+3206121 & 14.32 & 12.39 & 11.47 & M0 & L325 \\
\hline L108 & 2MASS J03443869+3208567 & 14.46 & 12.49 & 11.57 & M3.25 & L329 \\
\hline L115 & 2MASS J03442999+3209210 & 17.18 & 13.58 & 12.02 & M2.5 & L334 \\
\hline L116 & 2MASS J03442155+3210174 & 14.57 & 12.66 & 11.70 & M1.5 & L335 \\
\hline L119 & 2MASS J03442125+3205024 & 15.19 & 12.80 & 11.84 & $\mathrm{M} 2.5$ & L336 \\
\hline L123 & 2MASS J03442457+3203571 & 15.36 & 12.85 & 11.81 & M1 & L342 \\
\hline L124 & 2MASS J03435463+3200298 & 14.90 & 12.57 & 11.73 & M4.25 & L350 \\
\hline L125 & 2MASS J03442166+3206248 & 14.56 & 12.52 & 11.59 & M2.75 & L351 \\
\hline L128 & 2MASS J03442017+3208565 & 14.84 & 12.73 & 11.83 & M2 & L353 \\
\hline L140 & 2MASS J03443568+3203035 & 15.78 & 13.47 & 12.31 & M3.25 & L355 \\
\hline L142 & 2MASS J03435619+3208362 & 14.65 & 12.63 & 11.73 & M0 & L358 \\
\hline L145 & 2MASS J03444129+3210252 & 14.69 & 12.65 & 11.80 & M4.75 & L360 \\
\hline L146 & 2MASS J03444261+3206194 & 13.99 & 12.55 & 11.74 & M1 & L363 \\
\hline L149 & 2MASS J03443698+3208342 & 15.66 & 13.07 & 12.10 & M4.75 & L365 \\
\hline L153 & 2MASS J03444276+3208337 & 15.95 & 13.21 & 12.22 & M4.75 & L366 \\
\hline L156 & 2MASS J03440678+3207540 & 15.31 & 13.00 & 12.12 & M4.25 & L367 \\
\hline L158 & 2MASS J03444016+3209129 & 16.50 & 13.36 & 12.25 & M5 & L373 \\
\hline L159 & 2MASS J03444760+3210555 & 16.60 & 13.57 & 12.29 & M4.25 & L382 \\
\hline L160 & 2MASS J03440257+3201348 & 14.87 & 12.74 & 12.03 & M4.75 & L391 \\
\hline L163 & 2MASS J03441122+3208161 & 15.12 & 12.78 & 12.07 & M5.25 & L396 \\
\hline L165 & 2MASS J03443545+3208563 & 16.15 & 13.28 & 12.33 & M5.25 & L405 \\
\hline L166 & 2MASS J03444256+3210025 & 16.85 & 13.65 & 12.43 & M4.25 & L414 \\
\hline L167 & 2MASS J03444116+3210100 & 16.71 & 14.04 & 12.62 & M3 & L415 \\
\hline L168 & 2MASS J03443134+3210469 & 15.84 & 13.52 & 12.40 & M4.25 & L432 \\
\hline L169 & 2MASS J03441776+3204476 & 15.78 & 13.15 & 12.28 & M5.25 & L437 \\
\hline L174 & 2MASS J03440410+3207170 & 15.01 & 13.02 & 12.13 & M1.5 & L454 \\
\hline L182 & 2MASS J03441820+3209593 & 15.74 & 13.21 & 12.30 & M4.25 & L462 \\
\hline L187 & 2MASS J03440613+3207070 & 16.36 & 13.31 & 12.42 & M4.25 & L468 \\
\hline L190 & 2MASS J03442922+3201157 & 17.93 & 14.33 & 12.86 & M3.75 & L555 \\
\hline L192 & 2MASS J03442364+3201526 & 18.54 & 14.47 & 12.97 & M4.5 & L603 \\
\hline L194 & 2MASS J03442724+3210373 & 15.88 & 13.74 & 12.66 & M4.75 & L611 \\
\hline L198 & 2MASS J03443444+3206250 & 16.07 & 13.38 & 12.54 & M5.5 & L613 \\
\hline
\end{tabular}

Table 3

(Continued)

\begin{tabular}{|c|c|c|c|c|c|}
\hline ject & 2MASS ID & $I$ & $J$ & $H$ & SpT \\
\hline & 2MASS J03435721+3201337 & & & & M6.5 \\
\hline & 2MASS J03441810+3210534 & 18.24 & 16.04 & 13.90 & M0.75 \\
\hline & 2MASS J03442980+3200545 & 16.46 & 13.58 & 12.82 & M6 \\
\hline & 2MASS J03443030+3207426 & 17.16 & 14.01 & 12.70 & M3.5 \\
\hline & 2MASS J03442001+3206455 & 15.81 & 13.52 & 12.59 & M3.5 \\
\hline & 2MASS J03442894+3201378 & $\ldots$ & $\cdots$ & & M3.25 \\
\hline & 2MASS J03444303+3210151 & 16.07 & 13.54 & 12.64 & M5 \\
\hline & 2MASS J03444024+3209331 & 16.57 & 14.11 & 13.03 & M4.5 \\
\hline & $\cdots$ & 18.28 & 15.07 & 13.43 & M0.5 \\
\hline & 2MASS J03443551+3208046 & 16.30 & 13.66 & 12.78 & M5.25 \\
\hline & 2MASS J03444520+3201197 & $\cdots$ & $\cdots$ & $\cdots$ & M5.75 \\
\hline 37 & 2MASS J03442356+3209338 & 15.74 & 13.56 & 12.76 & M5 \\
\hline & 2MASS J03440770+3205050 & 16.71 & 14.01 & 12.98 & M4.5 \\
\hline & 2MASS J03442912+3207573 & 15.79 & 13.70 & 12.88 & M4.5 \\
\hline & 2MASS J03443165+3206534 & 16.10 & 13.58 & 12.82 & M5.5 \\
\hline & 2MASS J03435379+3207303 & 16.07 & 13.71 & 12.87 & M4.25 \\
\hline & 2MASS J03443569+3204527 & 16.10 & 13.70 & 13.01 & M5.75 \\
\hline & 2MASS J03435526+3207533 & 16.08 & 13.61 & 12.99 & M5.75 \\
\hline & 2MASS J03440362+3202341 & 16.44 & 13.54 & 12.88 & M5 \\
\hline & $\cdots$ & 16.04 & 13.73 & 12.93 & M4.75 \\
\hline & 2MASS J03440920+3202376 & 19.06 & 14.97 & 13.55 & M0 \\
\hline 77 & 2MASS J03443943+3210081 & 16.06 & 13.91 & 13.10 & M5 \\
\hline & 2MASS J03443103+3205460 & 16.75 & 14.03 & 13.18 & M5.5 \\
\hline & 2MASS J03444111+3208073 & 17.97 & 14.59 & 13.45 & M5.25 \\
\hline & 2MASS J03443886+3206364 & 16.60 & 13.98 & 13.26 & M6 \\
\hline & 2MASS J03443896+3203196 & 16.40 & 14.1 & 13.3 & M5 \\
\hline 01 & 2MASS J03442270+3201423 & 18.70 & 15.15 & 13.80 & M4.75 \\
\hline 02 & 2MASS J03442027+3205437 & 17.04 & 14.24 & 13.32 & M4.75 \\
\hline & 2MASS J03440442+3204539 & 16.60 & 14.06 & 13.38 & M5.75 \\
\hline & 2MASS J03442122+3201144 & & & & M4 \\
\hline 12 & 2MASS J03435508+3207145 & 16.80 & 14.12 & 13. & M6 \\
\hline 14 & 2MASS J03442256+3201277 & 18.80 & 15.13 & 13.80 & M5 \\
\hline & 2MASS J03441959+3202247 & 17.53 & 14.74 & 13.70 & M4.25 \\
\hline & 2MASS J03444522+3210557 & 17.14 & 14.56 & 13.65 & M5.75 \\
\hline & 2MASS J03443005+3208489 & 17.55 & 14.63 & 13.75 & M6 \\
\hline & 2MASS J03441558+3209218 & 17.64 & 14.57 & 13.85 & M7.5 \\
\hline 34 & 2MASS J03442666+3202363 & 16.88 & 14.42 & 13.69 & M5.75 \\
\hline & 2MASS J03444423+3208473 & 17.34 & 14.56 & 13.76 & M5.75 \\
\hline & 2MASS J03443237+3203274 & & & & M5.5 \\
\hline 42 & 2MASS J03444130+3204534 & 17.02 & 14.49 & 13.66 & M5 \\
\hline 50 & 2MASS J03441918+3205599 & 16.91 & 14.32 & 13.60 & M5.75 \\
\hline 51 & 2MASS J03442575+3209059 & 17.62 & 14.69 & 13.76 & M5.5 \\
\hline & 2MASS J03443814+3210215 & 16.87 & 14.46 & 13.70 & M6 \\
\hline & 2MASS J03443920+3208136 & 18.1 & 14.88 & 14.03 & M8 \\
\hline & 2MASS J03441276+3210552 & 16.79 & 14.61 & 13.92 & M5.5 \\
\hline & 2MASS J03444371+3210479 & 16.40 & 14.54 & 13.84 & M4.75 \\
\hline 63 & 2MASS J03441726+3200152 & 17.97 & 14.92 & 14.16 & M8 \\
\hline 65 & 2MASS J03441022+3207344 & 17.26 & 14.64 & 13.92 & M5.75 \\
\hline & 2MASS J03443501+3208573 & 17.33 & 14.84 & 14.05 & M4.75 \\
\hline & 2MASS J03435915+3205567 & 17.36 & 14.68 & 13.95 & M5.75 \\
\hline & 2MASS J03442798+3205196 & 17.18 & 14.84 & 14.14 & M5.5 \\
\hline 82 & 2MASS J03443095+3202441 & 18.95 & 15.48 & 14.47 & M5.5 \\
\hline & 2MASS J03444658+3209017 & 18.63 & 15.38 & 14.41 & M5.75 \\
\hline 96 & 2MASS J03440233+3210154 & 17.57 & 14.98 & 14.18 & M5.25 \\
\hline 05 & $\cdots$ & 18.34 & 15.20 & 14.48 & M8 \\
\hline 14 & 2MASS J03444428+3210368 & 17.68 & 15.41 & 14.68 & M5.25 \\
\hline 15 & 2MASS J03442997+3209394 & 18.43 & 15.20 & 14.36 & M6.5 \\
\hline & 2MASS J03444593+3203567 & 18.18 & 15.14 & 14.27 & M5.75 \\
\hline 37 & 2MASS J03435638+3209591 & 18.61 & 15.41 & 14.62 & M7.25 \\
\hline & 2MASS J03444157+3210394 & 17.81 & 15.38 & 14.61 & M5.75 \\
\hline & 2MASS J03442445+3201437 & 19.18 & 15.67 & 14.58 & M3 \\
\hline & 2MASS J03441106+3201436 & 20.55 & 16.53 & 15.42 & M8.25 \\
\hline & 2MASS J03444121+3206271 & 16.86 & 14.28 & 13.54 & M5.75 \\
\hline & 2MASS J03443341+3210314 & 19.95 & 16.33 & 15.61 & M8.5 \\
\hline & 2MASS J03443035+3209446 & 19.61 & 16.35 & 15.49 & M8 \\
\hline & 2MASS J03442685+3209257 & 19.80 & 16.86 & 16.01 & M8.25 \\
\hline
\end{tabular}


Table 3

(Continued)

\begin{tabular}{lccccc}
\hline \hline Object & 2MASS ID & $I$ & $J$ & $H$ & SpT \\
\hline L622 & $\ldots$ & 20.13 & 17.54 & 16.91 & M6 \\
L690 & 2MASS J03443638+3203054 & 20.02 & 16.62 & 15.78 & M8.75 \\
L703 & 2MASS J03443661+3203442 & 20.10 & 16.65 & 15.70 & M8 \\
L705 & $\ldots$ & 20.93 & 17.11 & 16.27 & M9 \\
L725 & $\ldots$ & 20.91 & 18.16 & 17.37 & M6 \\
L738 & $\ldots$ & 20.92 & 17.47 & 16.90 & M8.75 \\
L1683 & 2MASS J03441583+3159367 & $\ldots$ & $\ldots$ & $\ldots$ & M5.25 \\
L1684 & 2MASS J03442330+3201544 & 17.29 & 14.78 & 14.05 & M5.75 \\
L1889 & 2MASS J03442135+3159327 & $\ldots$ & $\ldots$ & $\ldots$ & $\ldots$ \\
L1925 & 2MASS J03440576+3200010 & $\ldots$ & $\ldots$ & $\ldots$ & M5.5 \\
L4011 & $\ldots$ & $\ldots$ & $\ldots$ & $\ldots$ & $\ldots$ \\
L4044 & $\ldots$ & 21.47 & 17.52 & 16.59 & M9 \\
L30003 & 2MASS J03435925+3202502 & $\ldots$ & $\ldots$ & $\ldots$ & M6 \\
\hline
\end{tabular}

Note. Identifications beginning with "L" are from the compilation of Luhman et al. (2003) and references therein, as are the photometry and spectral types.

Table 4

IC 348 Cluster Members Observed with the Hubble Space Telescope

\begin{tabular}{lcccc}
\hline \hline Object & $I$ & $J$ & $H$ & SpT \\
\hline L302 & 17.04 & 14.24 & 13.32 & M4.75 \\
L350 & 16.91 & 14.32 & 13.60 & M5.75 \\
L405 & 18.34 & 15.20 & 14.48 & M8 \\
L761 & 20.03 & 15.66 & 15.33 & M7 \\
L1434 & 21.11 & 18.39 & 17.44 & M6 \\
L4044 & 21.47 & 17.52 & 16.59 & M9
\end{tabular}

Notes. All information is from the compilation of Luhman et al. (2003), and references therein.

\subsection{Palomar 60 inch Telescope}

We used the P60 robotic telescope to observe BDs and VLMSs in the IC 348 cluster, as well as additional BDs in Upper Scorpius. Observations of IC 348 took place on a total of nine nights between 2008 November 17 and November 29. The chosen field center was R.A. $=03^{\mathrm{h}} 44^{\mathrm{m}} 19 \mathrm{~s} .7$, decl. $=+32^{\circ} 04^{\prime} 29^{\prime \prime} \mathrm{s}$ (J2000), but since the P60 system was at that time subject to tracking inaccuracies, this position shifted up to $45^{\prime \prime}$ throughout the run. We observed USco with the P60 during two different runs, from 2008 June 1 to 14, as well as 2009 May 14-30.

We chose exposure times of 240 or $300 \mathrm{~s}$ in the $i^{\prime}$ band to provide good sensitivity to the faint BDs without elongating the PSFs too much due to the lack of guiding. The established data reduction pipeline for the P60 performs basic calibrations, including bias subtraction, flatfielding, and fitting of the world coordinate system (Cenko et al. 2006). Although we obtained a series of sky flatfields, we determined that the domeflat images used by the pipeline were sufficient to correct interpixel sensitivity variations. Image alignment was also carried out with ease, since an accurate coordinate system was already superimposed on the calibrated images; we used the IRAF task wregister to complete this task.

\subsection{HST}

We used the HST WFC3 ultraviolet/visible (UVIS) CCD to observe pulsation candidates in IC 348. The UVIS channel is comprised of two chips, each with $4096 \times 2051$ pixels; since we observed in subarray mode, we used only one of these (UVIS1). Each pixel is $\sim 0$.'04 across, for a total subarray field of view of $\sim 81^{\prime \prime} \times 162^{\prime \prime}$.
Table 5

Objects in Upper Scorpius Observed as Part of the Pulsation Campaign

\begin{tabular}{lccclc}
\hline \hline Object & Telescope & $i$ & $J$ & SpT & Reference \\
\hline DENIS-P-J161050.0-221251.6 & CTIO 1.0 m & $\ldots$ & 12.80 & M5.5 & 1 \\
UScoJ16111705-2213088 & CTIO 1.0 m & $\ldots$ & 11.64 & M5 & 2 \\
SCH J16111711-22171749 & CTIO 1.0 m & 17.97 & 14.34 & M7.5 & 3 \\
UScoJ16113470-2219443 & CTIO 1.0 m & $\ldots$ & 13.24 & M5.75 & 2 \\
UScoJ16113784-2210275 & CTIO 1.0 m & $\ldots$ & 11.07 & M4 & 2 \\
SCH J16115737-22150691 & CTIO 1.0 m & 16.70 & 13.73 & M5 & 3 \\
SCH J16130306-19293234 & P60 & 16.75 & 13.45 & M5.5 & 3 \\
SCH J16132809-19245288 & P60 & 16.16 & 12.92 & M6 & 3 \\
SCH J16172504-23503799 & CTIO 1.0 m & 17.20 & 13.74 & M5 & 3 \\
SCH J16173105-20504715 & P60 & 16.49 & 13.03 & M7 & 3 \\
SCH J16174540-23533618 & CTIO 1.0 m & 17.44 & 14.05 & M6 & 3 \\
SCH J16181567-23470847 & CTIO 1.0 m & 16.18 & 12.42 & M5.5 & 3 \\
SCH J16182501-23381068 & CTIO 1.0 m & 17.19 & 13.72 & M5 & 3 \\
SCH J16183144-24195229 & P60 & 17.76 & 14.15 & M6.5 & 3 \\
SCH J16183620-24253332 & P60 & 14.75 & 12.03 & M4 & 3 \\
SCH J16185038-24243205 & P60 & 16.79 & 13.63 & M5 & 3 \\
\hline
\end{tabular}

Notes. References: (1) Martín et al. (2004); (2) Lodieu et al. (2011); (3) Slesnick et al. (2008)

Observations took place from 2011 January 29 to February 4, for just over seven hours of each day. Although HST is a space observatory, the Sun position and other observing constraints resulted in each block of observations ("visit") beginning at roughly the same time every day. Unfortunately much of visit 5 was compromised since the gyroscopic system failed and the field was lost for a number of hours. The viewing limits of HST are such that IC 348 objects may be observed for only 46 minutes of each 97 minute orbit. Therefore we designed exposure times to acquire as many images as possible per orbit, without exceeding the telescope's maximum data downlink rate. These varied among 128, 171, and $192 \mathrm{~s}$. All observations were carried out through the $\mathrm{F} 814 \mathrm{~W}$ filter, which is centered near $8030 \AA$ and similar to $I$ band.

HST/WFC3 data are processed by pipeline, which includes standard bias and flatfield calibration, as well as cosmic ray rejection. The MultiDrizzle program corrects for geometric distortion and optimally combines sets of three of four consecutive images, even for undithered data such as ours.

\section{PHOTOMETRY ROUTINES}

We performed aperture photometry on our target objects using a variety of aperture sizes and sky annulus widths and radii for background subtraction. Different approaches were chosen to produce ground and space-based photometry. For the P60 and CTIO $1.0 \mathrm{~m}$ data, we employed a variable-aperture method to optimize flux measurements in a variety of seeing conditions; see Cody \& Hillenbrand (2010) for a description of this technique. Both standard aperture photometry and image subtraction algorithms were tested. Typical apertures were 1-2 times the full width at half maximum (FWHM) PSF size, depending on object brightness, and sky annulus radii from 4.5-6 times the FWHM were used to subtract background. To locate photometric nonvariable reference stars, we identified suitable field objects. Typically, we found at least three to four references with photometric stability of several millimagnitudes over the full duration of each run.

For the HST/WFC3 images, apertures were fixed for each target and several different sizes were tested, from 2 to 8 pixel, as were sky annulus radii from 8 to 12 pixels and 11 to 16 pixels. Although results did not differ much, the best rms 

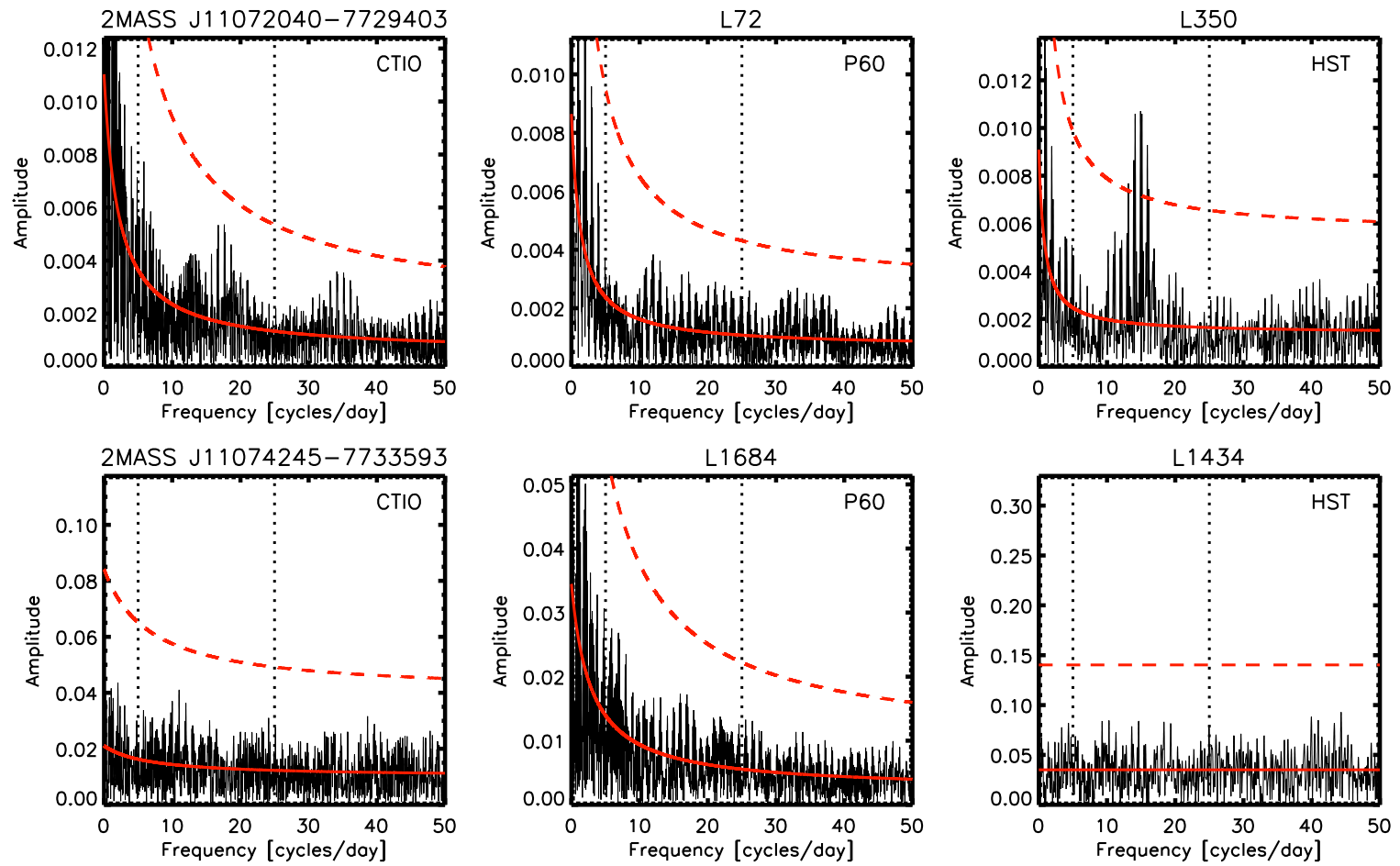

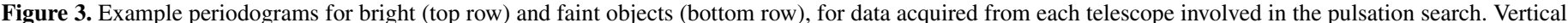

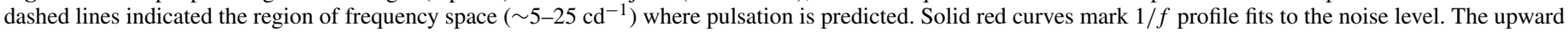

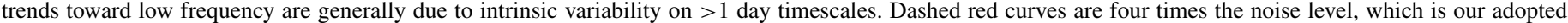

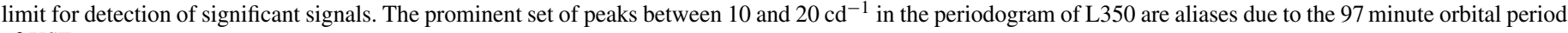
of HST.

(A color version of this figure is available in the online journal.)

light curve values were attained with the 6 pixel aperture and the sky annulus extending from 11 to 16 pixel. Although the Poisson errors are close to $0.001 \mathrm{mag}$ for several targets, the measured RMS light curve spreads are an order of magnitude larger, regardless of the type of photometry employed. This discrepancy suggests that the BDs and VLMSs observed with $H S T$ are all intrinsically variable.

The light curves produced for all fields were exclusively differential, since our primary interest in short-term flux variations makes photometric zero points irrelevant. By eye, the resulting time series from all telescopes display copious periodic and erratic variability, the latter of which is likely associated with accretion and circumstellar processes (see CH10 and the Appendix). To evaluate the performance of our photometric routines, we omitted variables with rms values of more than three standard deviations above the median trend as a function of magnitude. Careful attention to ground-based reduction procedures enabled us to reach a floor of several millimagnitude photometry at the bright end, comparable to the performance from the space observatories. In general, signal-to-noise, and hence photometric performance, decreased into the BD regime. We provide details on the data quality for individual clusters below in Section 5.

The final light curves were cleansed of artificial variability caused by, e.g., crossings of bad pixel columns and detector malfunctions. In the case of Cha I, a number of reference stars were unavailable on the first night of the run, since the two bottom quadrants of the Y4KCam CCD had failed. Not counting the first night, most Cha I light curves had 278 datapoints for the entire run. To recover additional photometry on the first night for stars in the top of the FOV, we performed image subtraction, which does not rely on reference stars. This resulted in an additional 26 datapoints for about half the targets. Light curves for the other clusters contained between 31 and 528 points.

\section{PERIODIC VARIABILITY CHARACTERIZATION}

Our main aim in analyzing the light curves of low-mass cluster members was to search for periodicities on the one to four hour timescales predicted for deuterium-burning instability. We produced Fourier transform periodograms (Deeming 1975), which display the amplitude of periodic signals as a function of frequency in units of cycles per day $\left(\mathrm{cd}^{-1}\right)$, along with the spectrum of noise in the light curve. Examples are displayed in Figure 3. We find that most display an exponential rise toward low frequencies, indicative of systematic effects (both night-tonight photometry shifts and intrinsic aperiodic stellar variability) on $>1$ day timescales in the light curve. Each periodogram is computed from a lower bound of $1 / T$, where $T$ is the total duration of observations, up to the Nyquist limit, or one half of the sampling rate (typically $\sim 70 \mathrm{~cd}^{-1}$ ).

True periodicities appear as localized peaks in the periodogram, and we identify them as statistically significant if they rise higher than a factor of 4.0 times the surrounding local noise level (see $\mathrm{CH} 10$ for further discussion on this requirement). We determine the noise profile by fitting a curve of form $A /(f+B)+C$ curve to the periodogram values as a function of frequency, $f$, with constants $A, B$, and $C$. This is displayed as the red curves in Figure 3 and confirms that the minimum detectable variability level at low frequencies is somewhat larger than amplitudes observable at higher frequencies (i.e., shorter timescales). 
We searched for periodicities in the periodograms corresponding to each light curve, paying particular attention to the $5-25 \mathrm{~cd}^{-1}$ range (i.e., periods of $1-4.8 \mathrm{hr}$ ) predicted for deuterium burning pulsation. If a period was found, we then removed the best-fit trend (based on multi-sine fits with the program Period04; Lenz \& Breger 2005) to produce a pre-whitened light curve. The search for few-hour periodicities was then carried out on this residual.

All targets were subjected to periodogram analysis. As a separate exercise, aperiodic variables were also identified; these "contaminants" are discussed in the Appendix. In the following sections, we describe the results of our period searches in each of the $\sigma$ Orionis, Cha I, IC 348, and Upper Scorpius regions.

\section{1. $\sigma$ Orionis}

With two nearly two-week observing campaigns on the CTIO $1.0 \mathrm{~m}$ telescope, we monitored over $150 \mathrm{VLMSs}$ and BDs in the $\sigma$ Orionis cluster. As detailed in $\mathrm{CH} 10$, we identified 38 periodically variable confirmed cluster members, along with 27 periodically variable candidate members. However, all but one of these variables had periods greater than $8 \mathrm{hr}$. The remaining single object (2MASS J053825570248370) had a period of $7.2 \mathrm{hr}$. This lack of detections in the one to four hour range was despite sensitivity to periods as short as 15 minutes, and amplitudes as low as several millimagnitudes down to a magnitude of $I=17$ (and as low as $1 \mathrm{mmag}$ at $I=14$ ). We therefore concluded that there are no signs of deuterium-burning pulsation in our $\sigma$ Ori data set. The full compilation of periodic variables, their phased light curves, and discussion of detection limits appears in $\mathrm{CH} 10$.

\subsection{Chamaeleon I}

Using data from the CTIO $1.0 \mathrm{~m}$ telescope, we produced and analyzed light curves for all 32 observed BD and VLMS members of the Cha I cluster. In the interest of fully mining the data set and potentially identifying new cluster members, we additionally performed photometry on all 1548 objects in the Cha I field that were bright enough for detection in individual Y4KCam images $(i \lesssim 22)$.

These additional light curves enabled an assessment of the photometric noise as a function of magnitude, as shown in Figure 4 . We have fit a trend to the cluster nonmembers, based on the expectations for Poisson and sky noise. Using the fit values as an indication of the magnitude-dependent photometric uncertainty, we have then selected variable objects via the $\chi^{2}$ test. The full collection of these light curves is presented and discussed in the Appendix. The floor of the rms distribution reaches $\sim 3$ mmag at an $I$-band magnitude of 14 , as shown in Figure 4 . Objects with $I<17$ display a photometric precision of $1 \%$ or better.

For each object in the sample, we carefully analyzed the periodograms for signs of periodicities on the few-hour timescales predicted for D-burning pulsation. Among the known cluster members, only four stars have estimated masses above $\sim 0.4 M_{\odot}$, and so most are candidates for the instability.

Based on the observing cadence of $600 \mathrm{~s}$, we are sensitive to periodic signals with frequencies as high as $72 \mathrm{~cd}^{-1}$ (i.e., $P=$ 20 minutes). The majority of light curves contain low-frequency variations, most of which are due to intrinsic erratic variability; these shape the exponential rise in periodogram amplitude at $<1 \mathrm{~cd}^{-1}$. Despite sensitivity to few-millimagnitude levels, we do not find any evidence for variability with periods less than $16 \mathrm{hr}$,

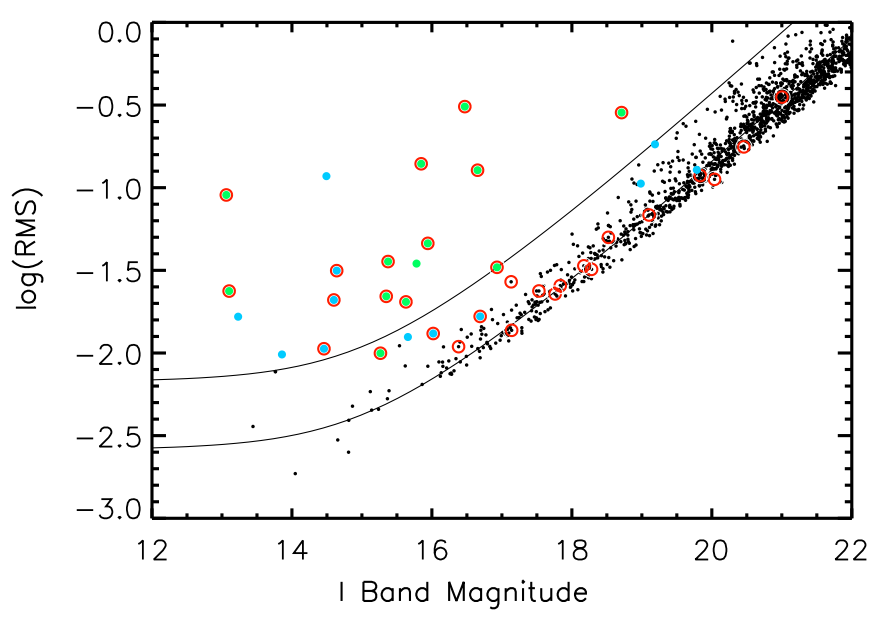

Figure 4. RMS spread of light curves over the 13-night duration of the Cha I observations, for periodic (blue) and aperiodic (green) variables. Confirmed cluster members appear as red circles. We plot the estimated total contributions from Poisson, mean sky level, and systematic noise, shifted upward by $0.24 \mathrm{dex}$ so as to match the median of the data (lower solid line). The curve corresponding to $99 \%$ probability of variability detection via the $\chi^{2}$ test appears above this.

(A color version of this figure is available in the online journal.)

apart from one field object that appears to be a main sequence pulsator. ${ }^{4} \mathrm{~A}$ few periodograms display low-level signals in the range where pulsation is expected, but none of these meet the 99\% significance level criteria, and we find most to be aperiodic variables. Furthermore, the light curves do not show clean trends when phased to these periods. We conclude that none of the 32 very-low-mass objects in the Cha I sample are periodic on one to five hour timescales, at least at or above the amplitude levels probed by the data.

On longer timescales, the final sample of variables contains 12 periodic objects in Cha I with clear variability. Observed and phased light curves for the cluster members among these are presented in Figure 5. We also identified periodic behavior among eight additional objects of unknown membership status. In most of these cases, the light curve shapes are characteristic of field pulsators or eclipsing binaries, and blue colors suggest locations in the background field. However, two relatively red objects with sinusoidal light curves may be bona fide Cha I members (2MASS J11073302-7728277 and 2MASS J11122675-7735183). The former (also known as CHXR 25) was classified by Luhman (2007) as a field dwarf, while the latter has no previous literature and would benefit from spectroscopic follow-up. The light curves for these new Cha I candidates are presented in Figure 6.

The measured properties for all variable sources in Cha I are listed in Table 6 . This includes the aperiodic variables uncovered with the $\chi^{2}$ test, as discussed in the Appendix. Of note, few Cha I members have been photometrically monitored previously, apart from a sample of $10 \mathrm{BDs}$ and VLMSs presented by Joergens et al. (2003). Of the five with periods reported in that work, none are redetected as periodic variables here.

$$
\text { 5.3. IC } 348
$$

In IC 348 the photometry includes only known cluster members, since high extinction in the region blocks our view of most background field stars. We were therefore unable to perform a full assessment of photometric precision based on

\footnotetext{
4 The star 2MASS J11105665-7733557 has a period of $3.3 \mathrm{hr}$ and amplitude 0.12 magnitudes in the $I$ band. However, its near-infrared colors lie well blueward of the young cluster sequence, implying that it is not a Cha I member.
} 

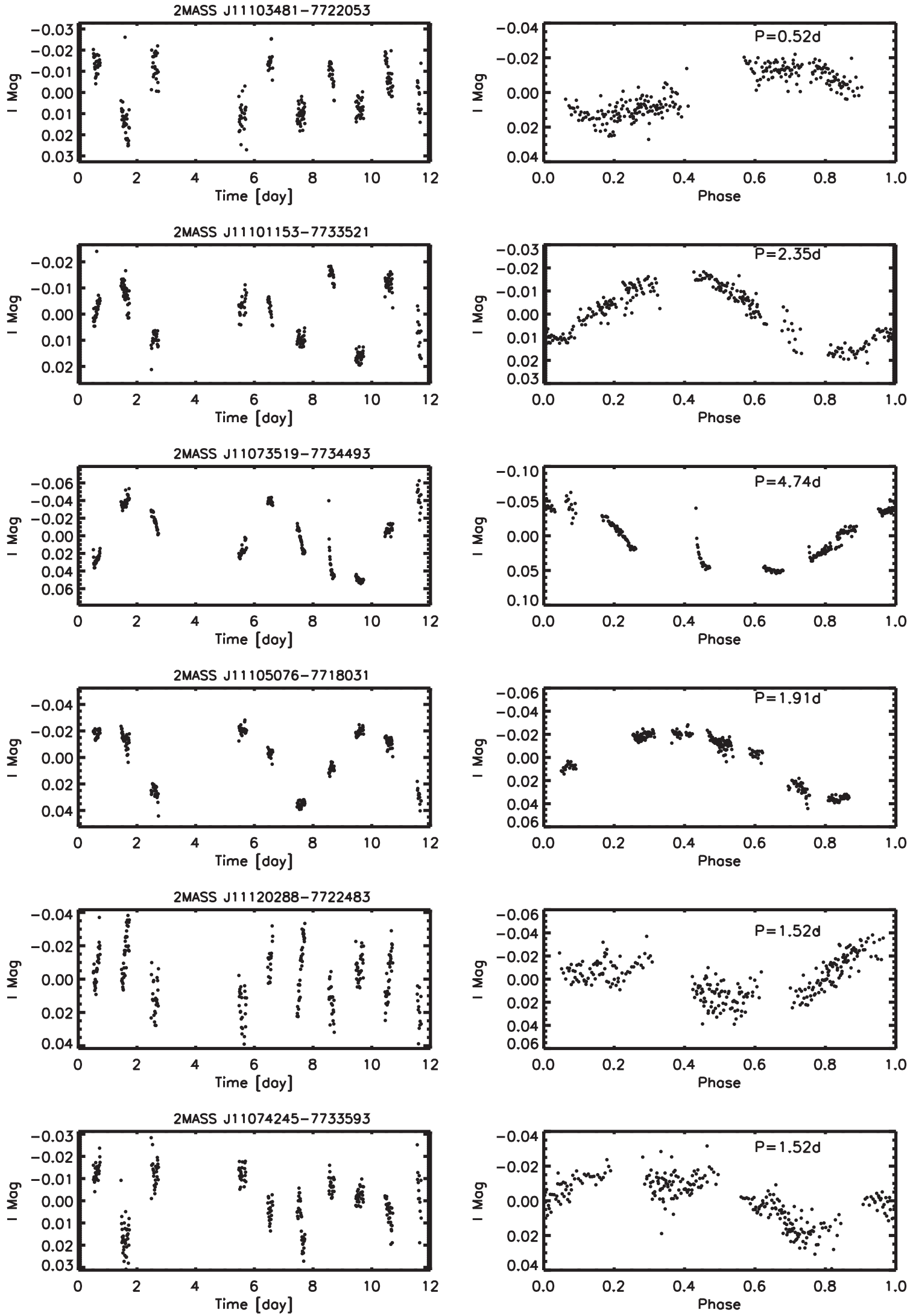

Figure 5. Differential light curves for very-low-mass Cha I members with detected periodic variability. The first column shows the original light curve, while the second is the light curve phased to the detected period. 

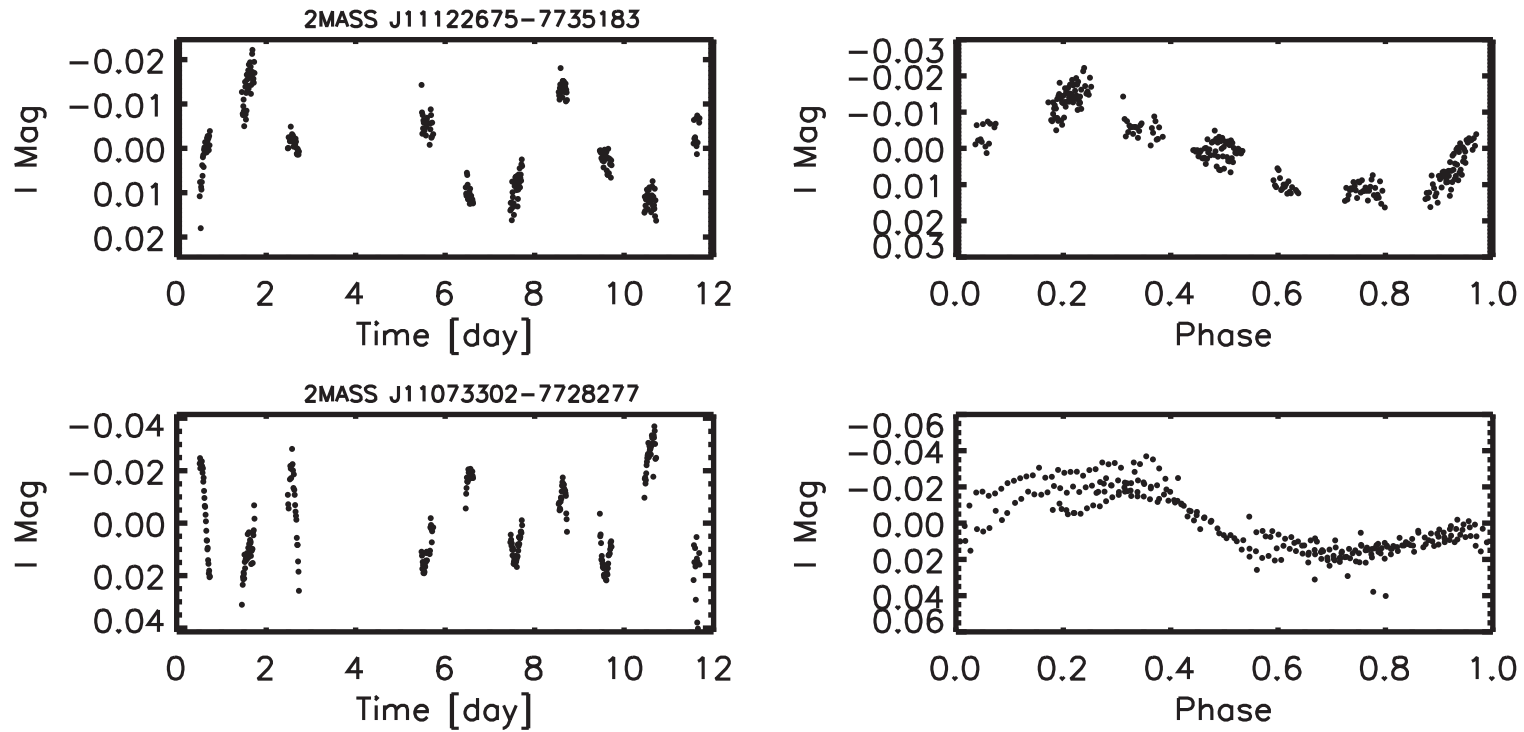

Figure 6. Light curves for new candidate Cha I members with detected periodic variability. The first column shows the original light curve, while the second is the light curve phased to the detected period.

Table 6

Cha I Objects with Detected Variability

\begin{tabular}{lcccccc}
\hline \hline Object & Variability Type & Period & Amplitude & RMS & Disk? & Member? \\
\hline 2MASS J11065906-7718535 & $\mathrm{A}$ & $\ldots$ & 0.405 & 0.090 & $\mathrm{Y}$ & $\mathrm{Y}$ \\
2MASS J11071668-7735532 & $\mathrm{A}$ & $\ldots$ & 0.405 & 0.090 & $\mathrm{Y}$ & $\mathrm{Y}$ \\
2MASS J11072040-7729403 & $\mathrm{A}$ & $\ldots$ & 0.105 & 0.022 & $\mathrm{~N}$ & $\mathrm{Y}$ \\
2MASS J11072988-7725017 & $\mathrm{P}$ & $2.28 \mathrm{~d}$ & 0.25 & 0.117 & $\ldots$ & $\mathrm{N}$ \\
2MASS J11073302-7728277 & $\mathrm{P}$ & $0.67 \mathrm{~d}$ & 0.0197 & 0.017 & $\mathrm{~N}$ & $\mathrm{M}$ \\
2MASS J11073519-7734493 & $\mathrm{P}$ & $4.74 \mathrm{~d}$ & 0.0478 & 0.031 & $\mathrm{~N}$ & $\mathrm{Y}$ \\
2MASS J11073686-7733335 & $\mathrm{A}$ & & 0.098 & 0.020 & $\mathrm{~N}$ & $\mathrm{Y}$ \\
2MASS J11074245-7733593 & $\mathrm{P}$ & $1.52 \mathrm{~d}$ & 0.0138 & 0.012 & $\ldots$ & $\mathrm{N}$ \\
2MASS J11075225-7736569 & $\mathrm{A}$ & $\ldots$ & 0.090 & 0.022 & $\mathrm{~N}$ & $\mathrm{Y}$ \\
2MASS J11082238-7730277 & $\mathrm{A}$ & $\ldots$ & 0.229 & 0.046 & $\mathrm{Y}$ & $\mathrm{Y}$ \\
2MASS J11083952-7734166 & $\mathrm{A}$ & $\ldots$ & 0.145 & 0.036 & $\mathrm{Y}$ & $\mathrm{Y}$ \\
2MASS J11085421-7732115 & $\mathrm{A}$ & $\ldots$ & 0.043 & 0.010 & $\mathrm{~N}$ & $\mathrm{Y}$ \\
CTIO J11093360-7731113 & $\mathrm{P}$ & $0.46 \mathrm{~d}$ & 0.1271 & 0.128 & $\ldots$ & $\mathrm{N}$ \\
2MASS J11094742-7726290 & $\mathrm{A}$ & $\ldots$ & 0.571 & 0.128 & $\mathrm{Y}$ & $\mathrm{Y}$ \\
2MASS J11094918-7731197 & $\mathrm{A}$ & $\ldots$ & 0.128 & 0.026 & $\mathrm{~N}$ & $\mathrm{Y}$ \\
2MASS J11101153-7733521 & $\mathrm{P}$ & $2.354 \mathrm{~d}$ & 0.0152 & 0.011 & $\mathrm{~N}$ & $\mathrm{Y}$ \\
2MASS J11103481-7722053 & $\mathrm{P}$ & 0.5229 & 0.0137 & 0.013 & $\mathrm{~N}$ & $\mathrm{Y}$ \\
2MASS J11105076-7718031 & $\mathrm{P}$ & $1.91 \mathrm{~d}$ & 0.0286 & 0.021 & $\mathrm{~N}$ & $\mathrm{Y}$ \\
2MASS J1105359-7725004 & $\mathrm{A}$ & $\ldots$ & 1.431 & 0.285 & $\mathrm{Y}$ & $\mathrm{Y}$ \\
2MASS J11105665-7733557 & $\mathrm{P}$ & $0.14 \mathrm{~d}$ & 0.1244 & 0.106 & $\ldots$ & $\mathrm{N}$ \\
CTIO J11111463-7737020 & $\mathrm{P}$ & $0.59 \mathrm{~d}$ & 0.1827 & 0.183 & $\ldots$ & $\mathrm{N}$ \\
2MASS J11120288-7722483 & $\mathrm{P}$ & $1.52 \mathrm{~d}$ & 0.0169 & 0.017 & $\mathrm{~N}$ & $\mathrm{Y}$ \\
2MASS J11120351-7726009 & $\mathrm{A}$ & $\ldots$ & 1.122 & 0.309 & $\mathrm{Y}$ & $\mathrm{Y}$ \\
2MASS J11122675-7735183 & $\mathrm{P}$ & $3.52 \mathrm{~d}$ & 0.0129 & 0.010 & $\mathrm{~N}$ & $\mathrm{M}$ \\
2MASS J11122971-7731045 & $\mathrm{A}$ & $\cdots$ & 0.171 & 0.035 & $\ldots$ & $\mathrm{M}$ \\
\hline
\end{tabular}

Notes. We list objects in the Cha I field with detected variability. "A" corresponds to aperiodic variability, while "P" is for periodic variability. The values listed in Column 4 are either the half amplitude for periodic sources or the peak-to-peak amplitude for aperiodic sources. In Column 5 we provide the rms light curve spreads in $I$-band magnitudes. The disk column indicates whether mid-infrared Spitzer data exhibits an excess; "-" indicates a lack of Spitzer photometry. Membership is based on previous censuses of Cha I; nonmembers ("N") have colors that are inconsistent with a position above the main sequence. "M" indicates a possible new cluster member based on the detected variability.

nonvariable field stars. However, a plot of rms light curves values versus magnitude (shown in Figure 7) provides a rough estimate of the performance for our P60 data set. Over the course of a single night, we reach a precision of $\sim 2$ mmag at $I=13$, and better than 0.01 mag down to $I \sim 17$. The rms values measured over the entire week-long duration of the light curves show considerably higher spreads in photometry, $\sim 1 \%$ or higher in most light curves. We attribute much of this to intrinsic variation of the stars on $>1$ day timescales.

We performed a period search analysis on all IC 348 cluster members observed with the P60 and HST. For the former, we are sensitive to periodicities on timescales from approximately 9 minutes to 12 days (resulting from a cadence of 270 s). Since we did not obtain data every night, our sensitivity to periods 


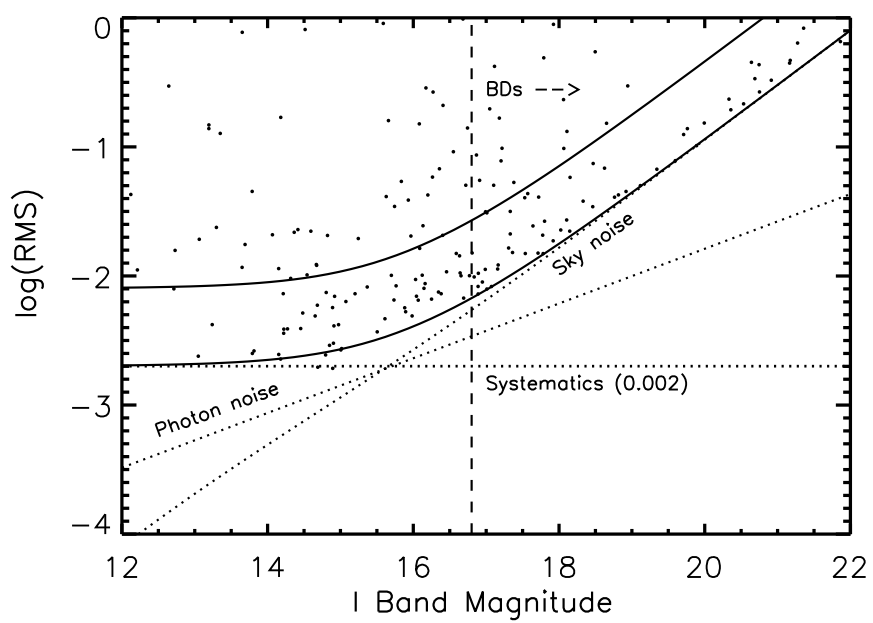

Figure 7. RMS spread of light curves for sources in IC 348, as measured over the course of a single night. All plotted objects are confirmed cluster members We show the estimated total contributions (solid line) from Poisson, mean sky level, and systematic noise. The upper solid curve corresponds to an estimate for $99 \%$ probability of variability detection. There is no clear boundary between variable and nonvariable sources here, unlike in Figure 4, which contains a mix of young cluster members and field stars.

of more than a few days is highly nonuniform. We therefore focused on the short periods predicted for D-burning pulsation.

We generated periodograms from the light curves of all 147 unsaturated objects in the ground-based P60 field, including 91 BDs and VLMSs. A large fraction of these display variability by eye, much of which is erratic and dominated by lowfrequency behavior. It is thus not surprising that many of the periodograms display excess power around 5-10 $\mathrm{cd}^{-1}$, which we attribute to aliasing, based on the window function, which shows a series of peaks centered around $8 \mathrm{~cd}^{-1}$. Further support for the spurious nature of these signals is that they do not reach the $99 \%$ significance level, and no coherent periodicity emerges when the light curve is phased.

In some cases, an obvious and statistically significant periodicity did appear on timescales of one day or more, suggesting rotation. When this occurred, we fit the overall trend and removed it from the light curve before searching for shorter timescale pulsation signals. In none of the IC 348 light curves did we uncover significant periodicities on the $1-5 \mathrm{hr}$ timescales indicative of pulsation.

Similar to the ground-based monitoring data, the HST time series are subject to aliasing. This effect is associated with the 97-minute orbital timescales and is seen prominently in the periodogram of object L350, shown in Figure 3. Omitting the frequency regions subject to aliasing, we have again searched for periodicities on one to five hour timescales, in hopes of detecting pulsation. In general, we find that the periodogram values are consistent with noise at the $1-5$ mmag level in the frequency range of interest $\left(5-25 \mathrm{~cd}^{-1}\right)$.

Two exceptions in the HST data set are the IC 348 BDs L761 and L1434. The former is periodically variable, as shown in Figure 8 . The period of 0.6 days is consistent with rotational modulation by one or more spots on its surface. L1434 is also variable (see Figure 9), but we are unable to determine whether it is periodic without a longer duration data set.

\subsection{Upper Scorpius}

As with the other young star regions, we produced discrete Fourier transforms to search for periodicities on a variety

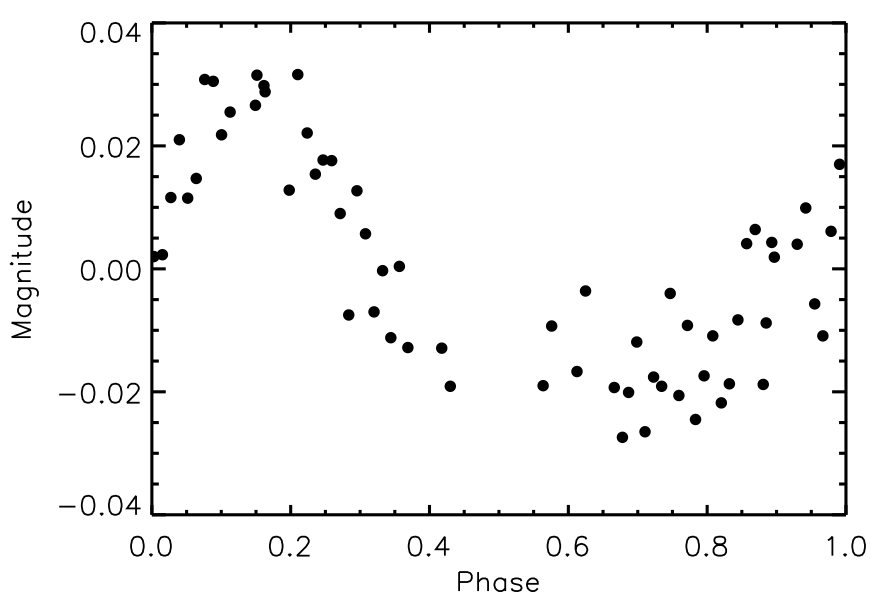

Figure 8. Phased light curve of the IC 348 object L761, observed with HST/ WFC3. This BD displays significant periodic variability on a timescale of 0.6 days.

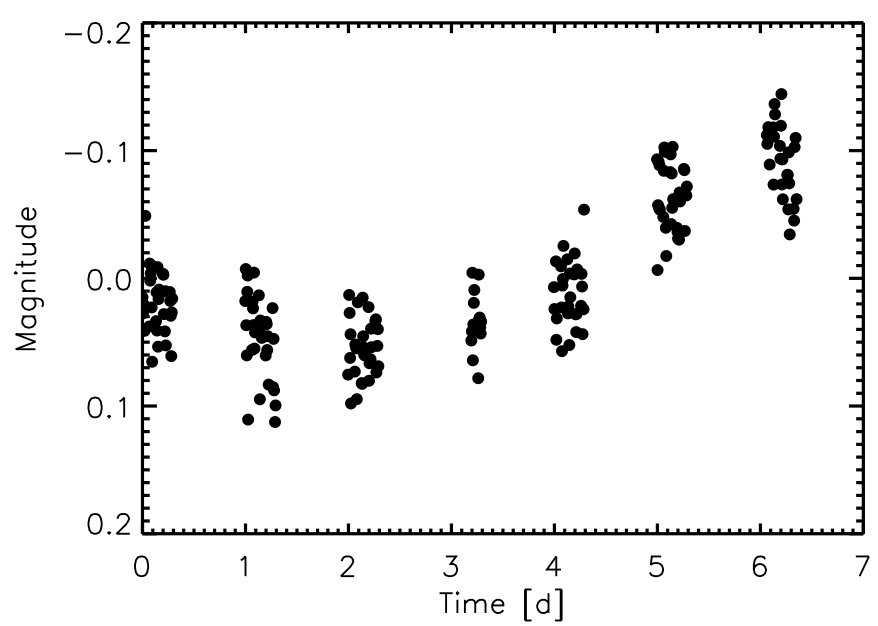

Figure 9. Light curve of the IC 348 object L1434, observed with HST/WFC3. This BD displays significant variability over the seven days that it was monitored.

of timescales in Upper Scorpius. Targets here were observed during a number of runs (see Table 1), and we are sensitive to periodicities as short as 10 minutes in each of these data sets. The longest period detectable varies with the length of each observing run and ranges from less than 2 days to 16 days. Since some of the runs only had six or fewer total nights, the associated periodograms have lower frequency resolution than the data sets on other clusters, and the search for periodicities is more susceptible to systematic effects. While some of the objects display variability on night-to-night timescales, we cannot accurately quantify all possible periodicities. We therefore focused exclusively on the search for pulsation at one to five hour periods. Detection of signals in this period range is feasible given the number of datapoints (30-550, depending on the run) and the fairly low photometric uncertainties of the BDs $(1 \%-3 \%)$.

We find that the data generally have sensitivity to signals in the few millimagnitude to $0.01 \mathrm{mag}$ amplitude range. Despite this, none of the objects in USco showed significant periodic variability. However, the light curves of a few displayed nightto-night variations that may be indicative of accretion or variable circumstellar extinction. 

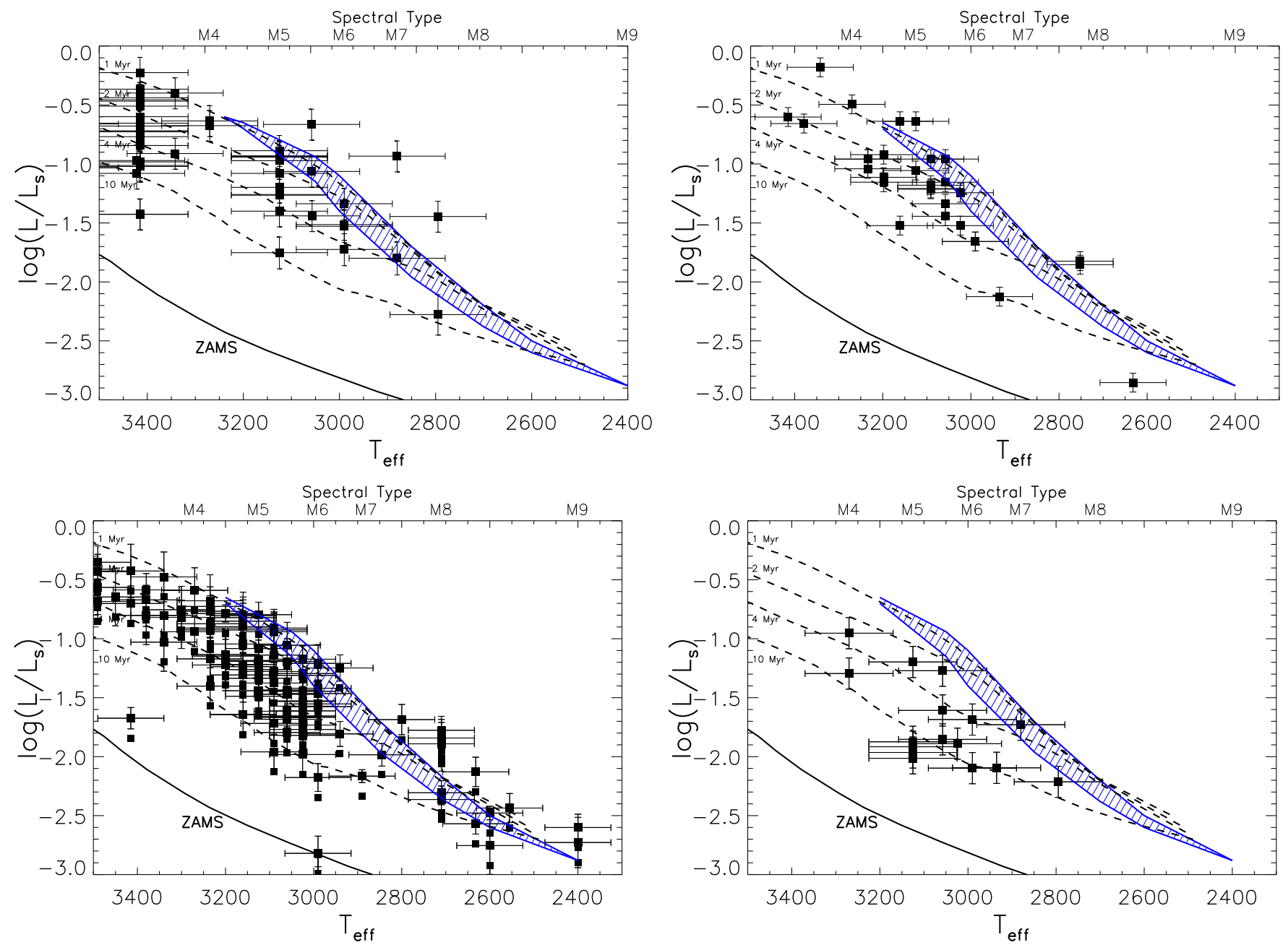

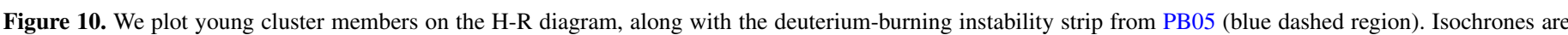

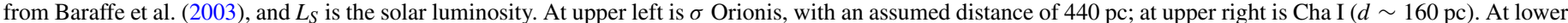

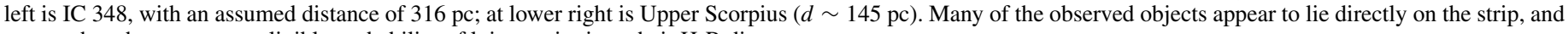
many others have a non-negligible probability of lying on it given their H-R diagram errors.

(A color version of this figure is available in the online journal.)

\section{NONDETECTION OF THE DEUTERIUM BURNING INSTABILITY}

With no periodicities observed in the one to four hour range for any of the clusters monitored, we now consider the implications of our nondetections. In each region, we assess the set of temperatures and luminosities in relation to the deuterium burning instability strip on the H-R diagram. The number of objects on or near the theoretically predicted region then sets the probability of detection, or lack thereof.

In $\sigma$ Orionis, our search for signs of deuterium burning oscillations among members was carried out with the CTIO $1.0 \mathrm{~m}$ and Spitzer telescopes, as detailed in Cody \& Hillenbrand (2010) and Cody \& Hillenbrand (2011), respectively. Within the uncertainties of cluster membership verification, there were approximately 40 objects with masses less than $\sim 0.1 M_{\odot}$. Of these, of order 15 had temperatures and luminosities overlapping the instability strip. We present the H-R diagram of observed Ori objects with available spectral types, for a distance of $440 \mathrm{pc}$, in Figure 10.

Our failure to detect short-period variability in $\sigma$ Ori is inconsistent with the predictions of PB05, a conclusion we further quantify in the next section. It is also at odds with previous reports of short-period variability in young BDs observed by Bailer-Jones \& Mundt (2001) and Zapatero Osorio et al. (2003).

Prior evidence for short-timescale variability was weaker (or nonexistent) in the other three clusters. To assess prospects for pulsation here, we performed the same H-R diagram evaluation as done for $\sigma$ Ori. In Cha I, four objects in our sample fall squarely on the instability strip when we convert spectral types to temperatures using the scale of Luhman et al. (2003). A further 24 have spectral types later than M4 and therefore may be burning deuterium and subject to pulsation. We plot the full sample of Cha I members in our field on the H-R diagram in Figure 10.

To estimate the number of our IC 348 targets that should be susceptible to the D-burning instability, we have plotted their positions on the H-R diagrams in Figure 10, assuming a distance of 316 pc. Many objects overlap the predicted D-burning pulsation strip. We also present the H-R diagram of observed USco objects in Figure 10. Of the 17 very-low-mass USco members observed, one lies squarely on the instability strip: SCH J16173105-20504715. However, its periodogram does not exhibit any significant signals in the region expected for pulsation. 

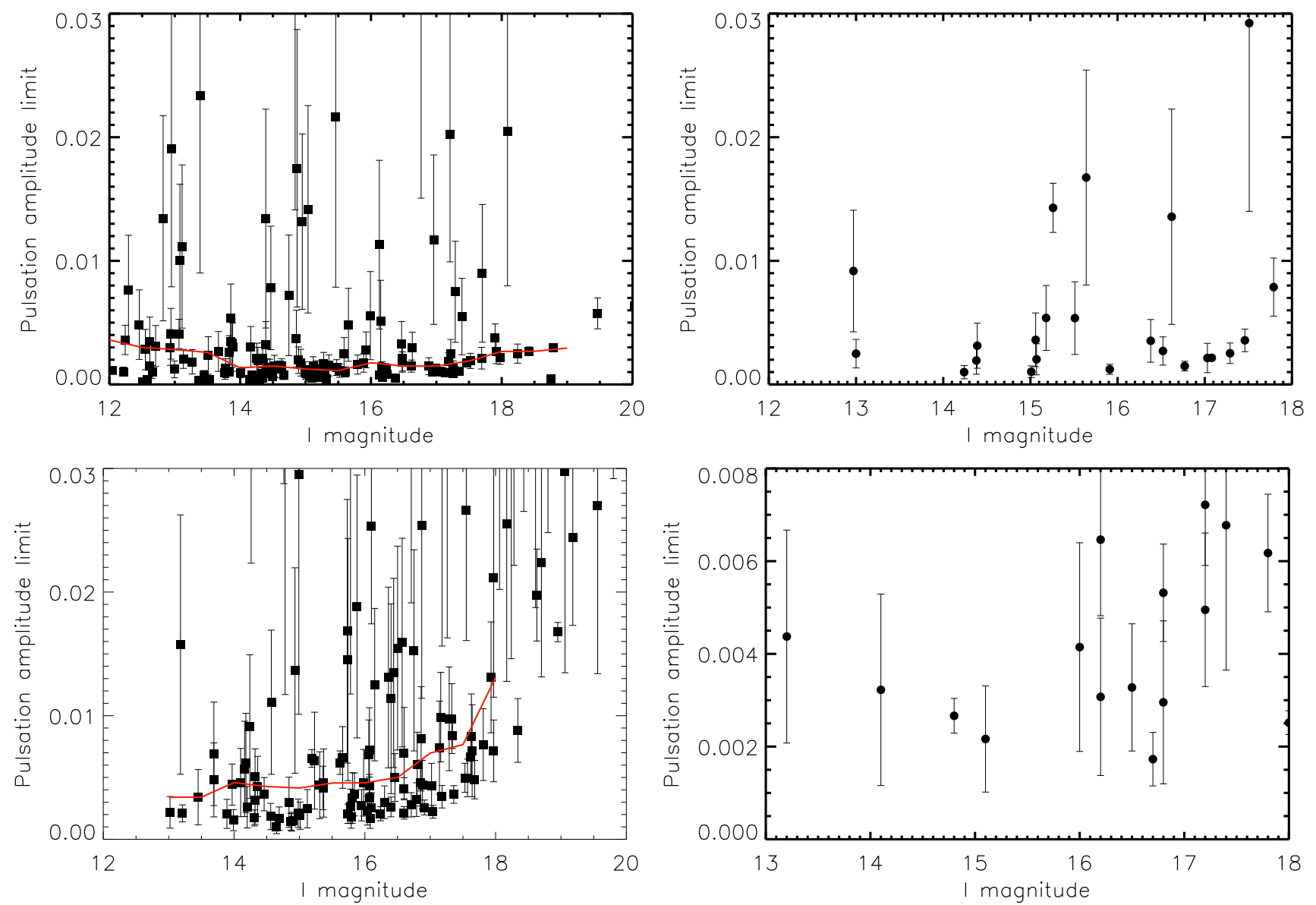

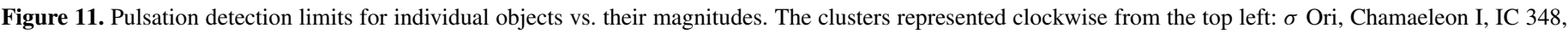

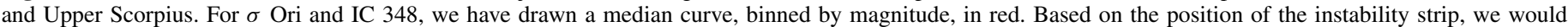

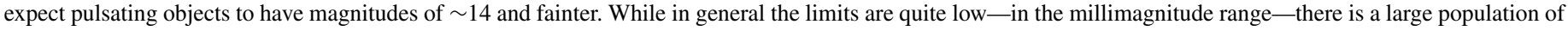
outliers in which high-amplitude intrinsic aperiodic introduced significant power into the higher frequency regions of the periodogram.

(A color version of this figure is available in the online journal.)

A number of other targets could lie on the strip if their $1 \sigma$ temperature and luminosity errors are considered, and this is true for each of the clusters examined. Below, we will use these uncertainties to assess the statistic likelihood of failing to observe pulsation in the overall monitoring data set.

\subsection{Statistical Evaluation of Pulsation Probabilities}

We uncovered many cases of periodic variability in the collected time series, over a wide range of timescales. Our detection of both rotation on $\sim 1-3$ day timescales in young cluster members and variability on hour timescales in background field pulsators and eclipsing binaries shows that our period detection algorithms are robust. Yet in the search for deuterium-burning pulsation, the data unanimously point to one conclusion: this instability is not present in young BDs and VLMSs above an amplitude of several millimagnitudes in the $I$ band.

One might argue that objects in our data set simply do not exhibit pulsation because they are not situated on the H-R diagram instability strip. However, the large sample size makes this possibility highly unlikely. To show how improbable the chances are that none of our sample have H-R diagram positions overlapping the instability strip, we consider temperature-luminosity probability distributions for each object. We take these to be twodimensional asymmetric Gaussians, normalized and centered at the adopted luminosities and temperatures. The Gaussian widths are given by the associated $1 \sigma$ uncertainties, which are shown in the H-R diagrams in Figure 10. The position of each target then corresponds to a probability that it is susceptible to pulsation, which we determine by integrating its distribution over the entire region of the instability strip. For objects on or very close to the strip, this value is at least $\sim 20 \%-25 \%$, whereas for the higher mass stars far from the strip it is close to zero. The probability that the position of a given object does not overlap with the instability strip is then 1.0 minus this quantity. The product of these values over all targets provides an estimate of the chance that no pulsators would be present in our sample.

We have performed this exercise for each of the clusters observed, and for alternate distances in cases where there is more than one possible value (IC 348 and $\sigma$ Ori). Since the instability strip runs nearly along isochrones and varies slowly in luminosity, changes in the adopted distance can have a significant impact on the number of objects expected to pulsate. We take all possibilities into consideration.

In Cha I, we determined an expectation value of 3-4 objects on the strip and find a probability of 0.015 that no object positions actually overlap it. Turning this number around, there is a nearly 99\% chance that at least one object should exhibit pulsation based on its position within the instability strip, assuming that the theoretical calculations underpinning it (PB05) do not suffer from gross systematic errors. Likewise in Cody \& Hillenbrand (2011), we found a 70\%-80\% chance that at least one of our $\sigma$ Ori targets observed in the infrared with Spitzer should exhibit 
pulsation. This is in contrast to the lack of short period variability in that data set.

USco does not have many targets overlapping the instability strip, and therefore the expectation is for only 1 or 2 objects to lie directly on it. In this region, we find a non-negligible probability of 0.22 that our sample did not include any pulsation candidates. For IC 348 , on the other hand, we expect $\sim 11$ objects on the strip and find a probability of $4 \times 10^{-6}$ that none are actually on it. This is assuming a distance of 316 pc Herbig (1998). If we instead assume a lower distance of 260 pc based on Hipparcos parallaxes (Scholz et al. 1999), then the expectation is similar: nine objects on the strip and a probability of $5 \times 10^{-5}$ that none are on it.

Finally, we have computed probabilities for $\sigma$ Ori. The precise number of pulsation candidates depends on the adopted distance, which is debated to be either $350_{-90}^{+120} \mathrm{pc}$, based on the Hipparcos parallax of $\sigma$ Ori $\mathrm{AB}$, or $440_{-30}^{+30}$ from main sequence fitting (Sherry et al. 2008). Assuming a cluster distance of $440 \mathrm{pc}$ (Sherry et al. 2008) we find that at least 4 targets are expected to be on the strip, with at most a 0.02 chance that none are. Substituting the alternate distance of $350 \mathrm{pc}$, we find nearly the same values $(3,0.06)$. The probabilities are upper limits since we do not have spectral types for part of the $\sigma$ Ori sample and hence cannot reliably place these objects on the H-R diagram.

In conclusion, we expect with high confidence to have observed deuterium-burning oscillations if it is present at observable amplitudes. We now quantify the overall detection limits by considering the power-law fit to the periodograms of each observed young cluster member. These curves, of form $A /(f+B)+C$ for frequency $f$ and constants $A, B$, and $C$, trace out the noise level as a function of frequency (see Figure 3 ). For each object analyzed, we take the fit values at $5 \mathrm{~cd}^{-1}(\sim 5 \mathrm{hr})$ and $25 \mathrm{~cd}^{-1}(\sim 1 \mathrm{hr})$ as representative of the $1 \sigma$ level above which no pulsation is observed. We display these values as a function of object magnitude in Figure 11 to illustrate the collective limit imposed by our entire data set.

The median amplitude limit is several millimagnitudes. Objects with high-amplitude aperiodic variability (see the Appendix) are exceptions, as they have excess periodogram noise which is intrinsic. The rest of our targets, however, have maximum amplitudes in the periodogram of at most 0.002-0.004 mag. This represents the threshold above which we detect no periodicities. We conclude that if deuterium-burning pulsation is present in any of our sources, then its amplitude must be below this level.

\section{CONCLUSION}

Despite exquisite photometric sensitivity, we have not detected signs of short-period variations in any of our young BD and VLMS targets. Although the theory of PB05 does not preclude very low amplitudes, we suspect that the failure to find pulsation is indicative of a physical damping mechanism operating within these objects. The convective timescale is over two orders of magnitude longer than the pulsation timescale, but it becomes quite short near the (sub)stellar surface layers. Neglect of the energy exchange between pulsation and convection may have led to overly optimistic predictions of mode amplitude growth. Indeed, models of this interaction in other types of stars have recently shown that convection can quench pulsation under some circumstances (Gastine \& Dintrans 2011).

To continue the search for pulsation and probe to lower amplitudes, future campaigns will need to produce extraordinarily high precision photometry. Data of this quality is available through the CoRoT mission, but only for stars brighter than $\sim 15$ th magnitude in the optical in the NGC 2264 cluster. K2, the successor mission to Kepler shows further promise as it will provide exquisite monitoring on hundreds of Upper Scorpius members.

Based on observations made with the NASA/ESA Hubble Space Telescope, obtained at the Space Telescope Science Institute, which is operated by the Association of Universities for Research in Astronomy, Inc., under NASA contract NAS 5-26555. These observations are associated with program 11610. A.M.C. thanks the CTIO Telescope Operations staff for help in carrying out observations. Observation time on SMARTS consortium facilities was awarded through the National Optical Astronomy Observatory, operated by the Association of Universities for Research in Astronomy, under contract with the National Science Foundation.

\section{APPENDIX \\ CONTAMINATION BY APERIODIC VARIABILITY}

In searching for periodicities among our young cluster targets, we encountered a number of light curves with rms values well above the median for field objects of similar magnitude. This aperiodic variability introduces low-frequency noise into the periodograms and raises the threshold over which we can detect pulsation signals. While this behavior may "contaminate" the pulsation search, it is interesting in its own right, as it offers insights into accretion and disk properties. We have assembled a collection of aperiodic variables in each of the clusters apart from USco, which did not have a sufficient sample size to mine non-periodic variables.

In general, we used plots of light curve rms versus magnitude (e.g., Figures 4 and 7) to select objects with variability at the 99\% confidence level (see CH10 Section 6 for a full description of detection procedures). In $\sigma$ Ori we uncovered 42 aperiodic variables with amplitudes from a few percent up to a full magnitude. Combining these with the periodically variable objects in that cluster, we found a total variable fraction of $69 \%$ for $\sigma$ Ori members ( $\mathrm{CH} 10)$.

Using the same detection approach in the Cha I field, we found 13 aperiodic variables, all but one of which are confirmed cluster members. The remaining object, 2MASS J11122971-7731045, has peak-to-peak variations of just over $0.1 \mathrm{mag}$ in the $I$ band. We provide the list of aperiodic variables in Table 6, and show their rms values in Figure 4. Their light curves are provided in Figure 12. Of note, three stars previously listed as periodic (Joergens et al. 2003) appear to be aperiodic, since they are detected as variable based on light curve rms but do not show distinct signals in their periodograms. These are 2MASS J11085421-7732115 (CHXR 78C), 2MASS J11075225-7736569 (Cha H $\alpha$ 3), and 2MASS J11083952-7734166 (Cha H $\alpha$ 6).

In the Cha I sample, the total variability fraction (including periodic objects) is between $53 \%$ and $69 \%$, depending on the membership status of several newly identified variable stars. This is statistically comparable to the fraction found in $\sigma$ Ori (CH10). The Cha I variability classification is divided into roughly equal proportions of periodic and aperiodic objects. Among the eight variables with no prior membership information, five have light curves and colors characteristic of field eclipsing binaries 

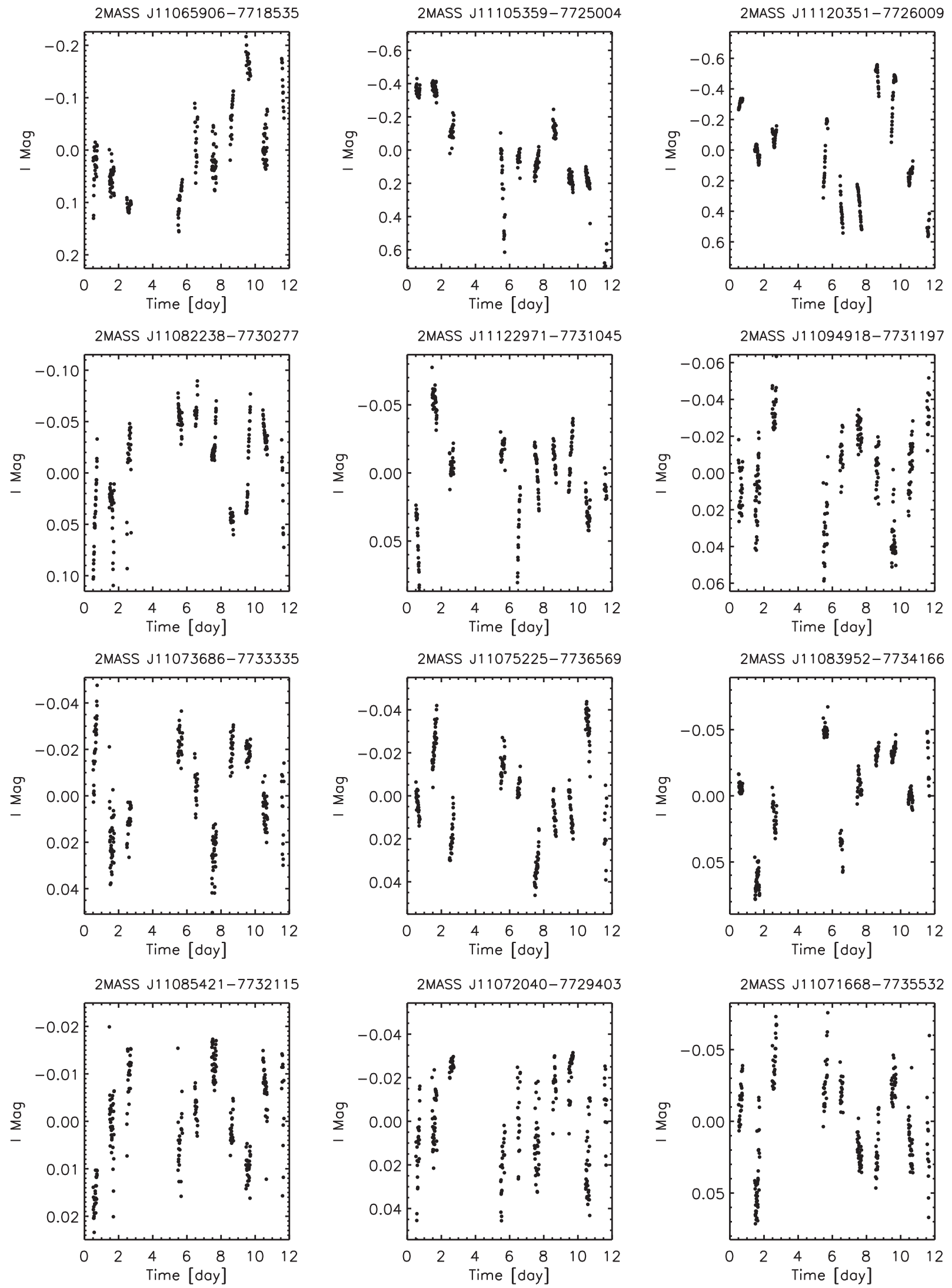

Figure 12. Light curves of very-low-mass Cha I members selected as aperiodic based on large $\chi^{2}$ values and lack of periodicities. Membership information is available in Table 6; 2MASS J11122971-7731045 is a new Cha I candidate. 

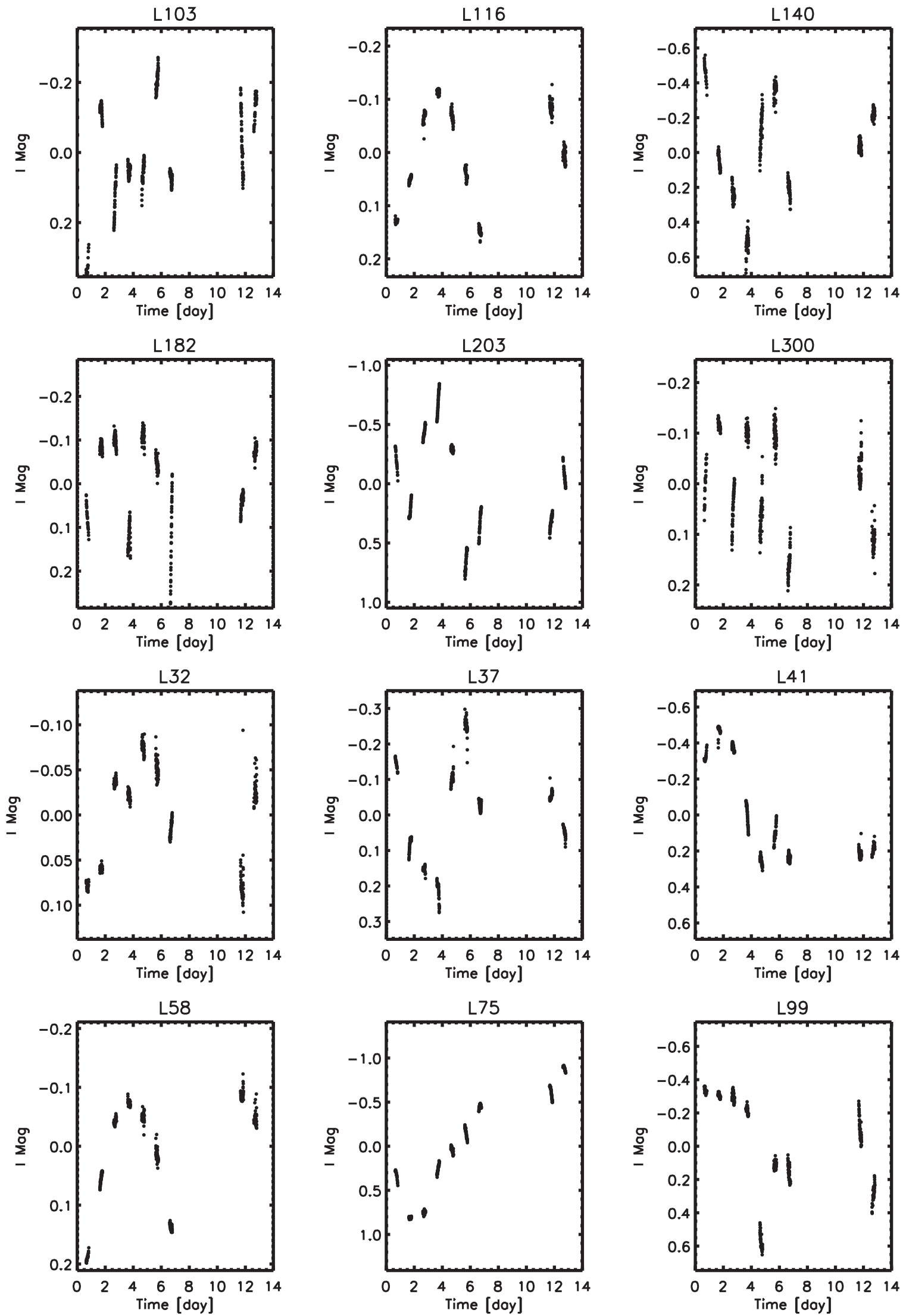

Figure 13. Light curves of IC 348 members selected by eye as being prominent variables. Ids are as in Table 3 . 


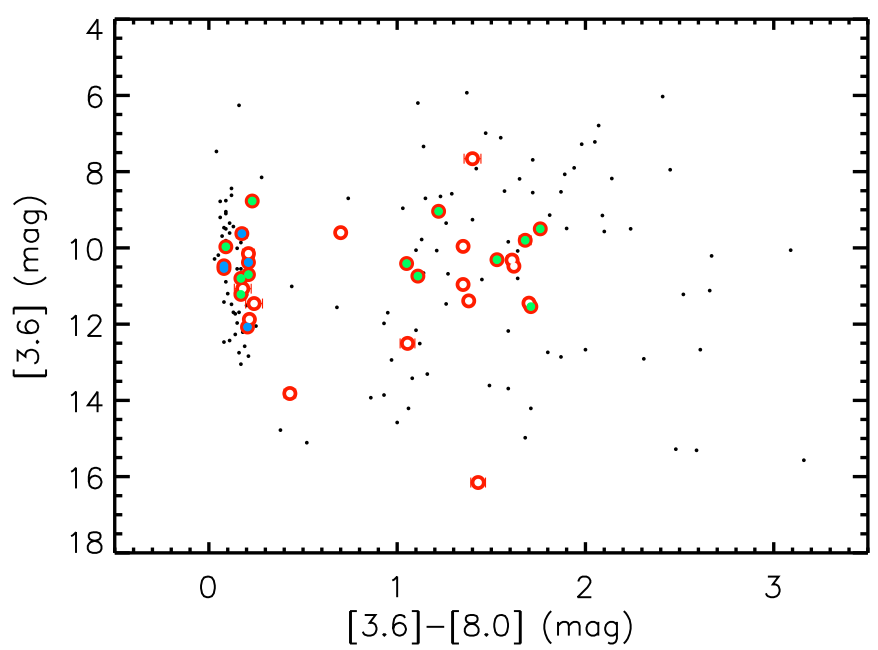

Figure 14. Spitzer photometry of likely Cha I members. Objects found in our photometric sample are marked with red circles, while those out of the fields are left as dots. Error bars are included but in many cases too small to see. Aperiodic variables detected in our photometry are overplotted as filled green circles, while periodic variables in our sample are marked by filled blue circles. The nearly vertical cluster of objects near [3.6]-[8.0] $=0$ is the sequence of colors and magnitudes pertaining to bare photospheres.

(A color version of this figure is available in the online journal.)

or pulsators. Three may be new members, and we note these in Table 6.

In the IC 348 sample, there were no nonvariable field stars available for determination of the photometric uncertainty as a function of magnitude. As a result, we could not calculate accurate $\chi^{2}$ values for the light curves from this cluster. However, a rough cutoff for rms values indicative of variability (i.e., upper line in Figure 7) suggests that there are a number high-amplitude variables. We examined the light curves of objects above the upper curve in Figure 7 and removed from consideration those for which artificial systematic effects (i.e., tracking and flatfielding errors) appeared to cause a high RMS. We are left with nine IC 348 objects with strong variability; these light curves are depicted in Figure 13. Their identification numbers, from Luhman et al. (2003), are provided above each panel.

Beyond identification of the aperiodic variables in our data set, we can assess correlations between their flux behavior and the presence of circumstellar disks. We cross-referenced our photometric samples in $\sigma$ Ori and Cha I with those of Luhman et al. (2008), and Luhman \& Muench (2008), which provide Spitzer Infrared Array Camera (IRAC; 3.6-8.0 $\mu \mathrm{m}$ ) and in some cases Multiband Imaging Photometer for Spitzer (MIPS; $24 \mu \mathrm{m}$ ) photometry. In $\sigma$ Ori, we find that 133 of 153 confirmed or candidate members in our time series data set have Spitzer photometry, including 57 of 65 cluster periodic variables. For the Cha I sample, all 37 cluster members monitored in the $i$ band have available IRAC measurements, and in many cases, MIPS data in addition. While there is Spitzer data available for the IC 348 cluster as well, we have not included it in the analysis here since extensive comparison of photometric periods with infrared excess was already carried out by Cieza \& Baliber (2006). Furthermore, our own IC 348 photometry is difficult to cleanly separate into the periodic and aperiodic categories due to the systematics resulting from the lack of tracking on the P60 telescope.

We display in Figure 14 the distribution of Spitzer/IRAC 3.6-8.0 $\mu \mathrm{m}$ colors for all objects in our Cha I data set with available infrared photometry; the equivalent plot for $\sigma$ Orionis

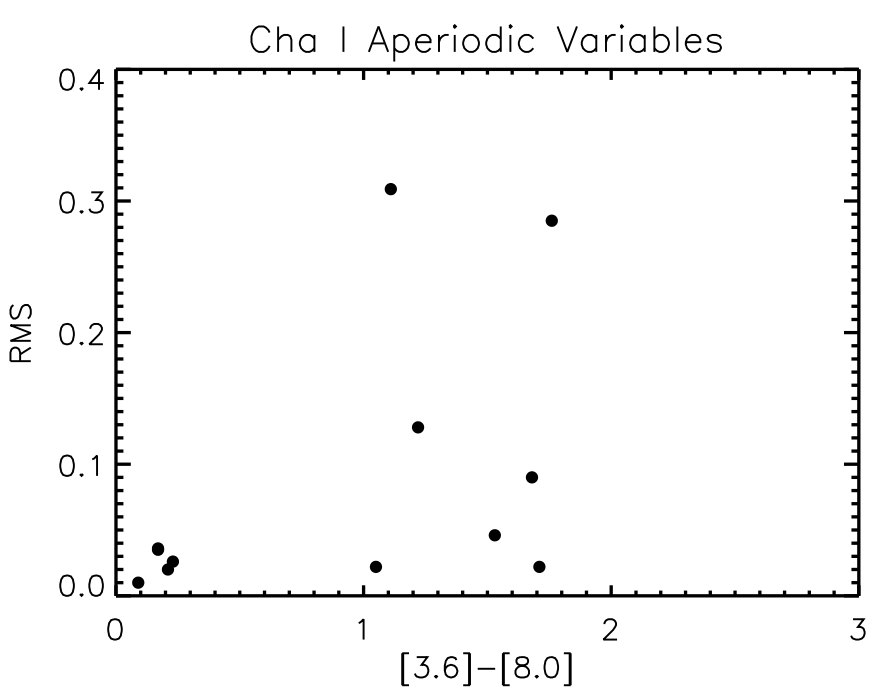

Figure 15. Spitzer [3.6]-[8.0] color vs. light curve I-band rms value for our aperiodic variables in Cha I.

is shown in Figure 13 of $\mathrm{CH} 10$. As seen in these diagrams, the samples split relatively cleanly into two groups, with the narrower blue sequence near [3.6]-[8.0] $=0$ representing bare photosphere colors. The cloud of objects with [3.6]-[8.0] colors between 1 and 2 is indicative of infrared excesses signifying the presence of a dusty disk. While the sequence of photospheric colors is fairly well defined, several ambiguous objects lie between 0.3 and $0.7 \mathrm{mag}$. We have therefore chosen a somewhat conservative disk selection criteria of [3.6]-[8.0] $>0.7$ (e.g., Cieza \& Baliber 2007) so as to omit these objects from the disk sample.

In total, we identify 21 disk-bearing Cha I members with both photometry from our campaign and Spitzer colors indicative of disks. The resulting disk fraction in our sample is $57 \pm 9 \%$. We find that our disk identification is entirely consistent with that of Luhman et al. (2008) and Luhman \& Muench (2008) (based on the same Spitzer data). The full listing of disk classifications is provided in Table 6 .

In Figure 14 we have distinguished variable objects (blue for periodic; green for aperiodic) from the nonvariables (white) in the Spitzer/IRAC color-magnitude diagram. In the small Cha I sample, none of the periodic variables has an infrared excess suggestive of a disk. As was the case with $\sigma$ Orionis (CH10), we can associate disks with the majority of aperiodic variables in our sample and lack of a disk with most of the periodic variables. But a number of objects do not fit these scenarios.

Five Cha I members display aperiodic variability but no sign of infrared excess in the Spitzer data. This curious small population of objects with rms values $(\sim 0.01-0.03 \mathrm{mag})$ much lower than the other aperiodic variables and have $\mathrm{H} \alpha \mathrm{pEW}$ and [3.6]-[8.0] values suggesting absence of accretion or an associated disk. In addition to light curves in which variability is clearly obvious by eye, these objects have $\chi^{2}$ values high enough that their status as variables is not in doubt. All but one have $\chi^{2}>4.5$, or less than $10^{-5}$ probability that the light curve trends arose by chance; the remaining object (2MASS $\mathrm{J} 05383922-0253084$ ) has a $\chi^{2}$ value of 2.85 , or an estimated $0.4 \%$ probability that its light curve behavior is explained by noise. We show in Figure 15 the rms and infrared colors for Cha I members; Figure 15 of $\mathrm{CH} 10$ illustrates another such cluster of low-amplitude aperiodic variables with no signs of circumstellar disks. 
A similar phenomenon was observed in the IC 348 cluster, in which a number of weak T Tauri stars (i.e., weak $\mathrm{H} \alpha$ ) were found to be erratic variables by Littlefair et al. (2005). These results bring into question our ability to determine which cluster members are truly surrounded by disk material, which ultimately affects the analysis of rotation and possible disk locking. It appears from these light curves that a percentage of young objects retain enough gas and/or dust beyond the time that we would expect their disks to be fully cleared based on infrared observations. Alternatively, we may be viewing rapid evolution of magnetic spot features on the stellar surface.

\section{REFERENCES}

Bailer-Jones, C. A. L., \& Mundt, R. 2001, A\&A, 367, 218

Baraffe, I., Chabrier, G., Barman, T. S., Allard, F., \& Hauschildt, P. H. 2003, A\&A, 402, 701

Barrado y Navascués, D., Béjar, V. J. S., Mundt, R., et al. 2003, A\&A, 404, 171 Barrado y Navascués, D., Zapatero Osorio, M. R., Béjar, V. J. S., et al. 2001, A\&A, 377, L9

Beck, P. G., Montalban, J., Kallinger, T., et al. 2012, Natur, 481, 55

Béjar, V. J. S., Martín, E. L., Zapatero Osorio, M. R., et al. 2001, ApJ, 556, 830

Béjar, V. J. S., Zapatero Osorio, M. R., \& Rebolo, R. 1999, ApJ, 521, 671

Béjar, V. J. S., Zapatero Osorio, M. R., \& Rebolo, R. 2004, AN, 325, 705

Burningham, B., Naylor, T., Littlefair, S. P., \& Jeffries, R. D. 2005, MNRAS, 356,1583

Caballero, J. A. 2008, A\&A, 478, 667

Caballero, J. A., Béjar, V. J. S., Rebolo, R., et al. 2007, A\&A, 470, 903

Caballero, J. A., Béjar, V. J. S., Rebolo, R., \& Zapatero Osorio, M. R. 2004, A\&A, 424, 857

Cenko, S., Fox, D. B., Moon, D., et al. 2006, PASP, 118, 1396

Cieza, L., \& Baliber, N. 2006, ApJ, 649, 862

Cieza, L., \& Baliber, N. 2006, ApJ, 649, 862

Cieza, L., \& Baliber, N. 2007, ApJ, 671, 605

Cody, A. M., \& Hillenbrand, L. A. 2010, ApJS, 191, 389

Cody, A. M., \& Hillenbrand, L. A. 2011, ApJ, 741, 9

Cohen, R. E., Herbst, W., \& Williams, E. C. 2004, AJ, 127, 1602

Dawson, P., Scholz, A., \& Ray, T. P. 2011, MNRAS, 418, 1231

Deeming, T. J. 1975, Ap\&SS, 36, 137

Franciosini, E., Pallavicini, R., \& Sanz-Forcada, J. 2006, A\&A, 446, 501

Gastine, T., \& Dintrans, B. 2011, A\&A, 528, A6
Guenther, D. B., Kallinger, T., Zwintz, K., et al. 2009, ApJ, 704, 1710

Herbig, G. 1998, ApJ, 497, 736

Hernández, J., Hartmann, L., Megeath, T., et al. 2007, ApJ, 662, 1067

Joergens, V., Fernández, M., Carpenter, J. M., \& Neuhäuser, R. 2003, ApJ, 594,971

Kenyon, M. J., Jeffries, R. D., Naylor, T., Oliveira, J. M., \& Maxted, P. F. L. 2005, MNRAS, 356, 89

Kurtz, D. W., Saio, H., Takata, M., et al. 2014, MNRAS, 444, 102

Lebreton, Y., \& Goupil, M.-J. 2014, A\&A, 569, 21

Lenz, P., \& Breger, M. 2005, CoAst, 146, 53

Littlefair, S. P., Naylor, T., Burningham, B., \& Jeffries, R. D. 2005, MNRAS, 358,341

Lodieu, N., Dobbie, P. D., \& Hambly, N. C. 2011, A\&A, 527, 24

Lodieu, N., Zapatero Osorio, M. R., Rebolo, R., Martín, E. L., \& Hambly, N. C. 2009 , A\&A, 505, 1115

Luhman, K. L. 2004, ApJ, 602, 816

Luhman, K. L. 2007, ApJS, 173, 104

Luhman, K. L., Allen, L. E., Allen, P. R., et al. 2008, ApJ, 675, 1375

Luhman, K. L., Hernández, J., Downes, J. J., Hartmann, L., \& Briceño, C. 2008, ApJ, 688, 362

Luhman, K. L., Lada, E. A., Muench, A. A., \& Elston, R. J. 2005, ApJ, 618,810

Luhman, K. L., \& Muench, A. A. 2008, ApJ, 684, 654

Luhman, K. L., Stauffer, J. R., Muench, A. A., et al. 2003, ApJ, 593, 1093

Martí, B. L., Eislöffel, J., Scholz, A., \& Mundt, R. 2004, A\&A, 416, 555

Martín, E. L., Delfosse, X., \& Guieu, S. 2004, AJ, 127, 449

Muench, A. A., Lada, C. J., Luhman, K. L., Muzerolle, J., \& Young, E. 2007, AJ, 134,411

Mužić, K., Scholz, A., Geers, V., Fissel, L., \& Jayawardhana, R. 2011, ApJ, 732,86

Palla, F., \& Baraffe, I. 2005, A\&A, 432, L57

Pecaut, M. J., Mamajek, E. E., \& Bubar, E. J. 2012, ApJ, 746, 154

Preibisch, T., Brown, A. G. A., Bridges, T., Guenther, E., \& Zinnecker, H. 2002, AJ, 124, 404

Scholz, A., \& Eislöffel, J. 2004, A\&A, 419, 249

Scholz, R.-D., Brunzendorf, J., Ivanov, G., et al. 1999, A\&AS, 137, 305

Sherry, W. H., Walter, F. M., \& Wolk, S. J. 2004, AJ, 128, 2316

Sherry, W. H., Walter, F. M., Wolk, S. J., \& Adams, N. R. 2008, AJ, 135, 1616

Slesnick, C. L. 2008, PhD thesis, California Institute of Technology

Slesnick, C. L., Hillenbrand, L. A., \& Carpenter, J. M. 2008, ApJ, 688, 377

Zapatero Osorio, M. R., Caballero, J. A., Béjar, V. J. S., \& Rebolo, R. 2003, A\&A, 408, 663

Zwintz, K. 2008, ApJ, 673, 1088

Zwintz, K., Fossati, L., Ryabchikova, T., et al. 2014, Sci, 345, 550 